\title{
Evolution of solutions for dipolar bodies in Thermoelasticity without energy dissipation
}

\author{
Marin Marin and Ibrahim Abbas
}

\begin{abstract}
The aim of our paper is the study of the spatial evolution of vibrations in the context of Thermoelasticity without energy dissipation for dipolar bodies. Once we get an a priori estimate for the amplitude of the vibration, which are assumed being harmonic in time, it is possible to predict some spatial decay or growth properties for the amplitude, provided the frequency of vibration is greater than a certain critical value.
\end{abstract}

\section{Introduction}

We consider a right prismatic cylinder composed of a physically dipolar thermoelastic body. The cylinder is free of loads on lateral surface (no body force, no dipolar body force and no heat supply), while on the base of cylinder are prescribed a time-dependent displacement, a dipolar displacement and a thermal displacement, which are harmonic in time. We associate a measure of Toupin type to the corresponding steady-state vibration and we will obtain a spatial decay estimate by assuming that the exciting frequency is lower to a certain critical frequency.

It is known that the theory of thermoelastic material behavior without energy dissipation possesses the following properties: the heat flow, in contrast to that in classical thermoelasticity characterized by the Fourier law, does not

Key Words: dipolar bodies; energy dissipation; harmonic vibration; thermoelasticity

2010 Mathematics Subject Classification: Primary ; Secondary .

Received:2.06.2015

Revised:30.07.2015

Accepted:2.10.2015 
involve energy dissipation. In addition, a constitutive equation for an entropy flux vector is determined by the same potential function which also determines the stress, and it permits the transmission of heat as thermal waves at finite speed.

The origin of the theories of dipolar bodies goes back to Mindlin which in his famous paper [17] proved that the linear theory of a micropolar continuum is a special case of the theory of dipolar media.

There were several motivations for the extension of the classical theory to dipolar. For instance, the classical theory was not able to predict the size effect experimentally observed in problems which had a geometric length scale comparable to material's microstructural length, such as the grain size in a polycrystalline or granular aggregate. Also, the apparent strength of some materials with stress concentrators such as holes and notches is higher for smaller grain size; for a given volume fraction of dispersed hard particles, the strengthening of metals is greater for smaller particles; the bending and torsional strengths are higher for very thin beams and wires.

The theories of dipolar bodies are quit sufficient for a large number of solid mechanics applications. Because the system of governing equations and conditions for the thermoelasticity of dipolar bodies is more complicated, it is neccesary a new approach for the boundary value problem in this context.

In specific literature, there are many studies for describing the heat conduction which are also called theories of second sound, where the flow of heat is modelled with finite propagation speed, in contrast to the classical model based on the Fourier's law leading to infinite propagation speed of heat signals. Several articles of this kind are analyzed in the paper [1] by Chandrasekharaiah.

It is believed that Green and Naghdi [7] have obtained the first results in the thermoelastic theory without energy dissipation, where they introduce the so-called thermal displacement related to the common temperature and uses a general entropy balance as postulated in Green and Naghdi [8]. By the procedure of Green and Naghdi, the reduced energy equation is regarded as an identity for all thermodynamical processes and places some restrictions on the functional forms of the dependent constitutive variables. The theory is illustrated in detail in the context of flow of heat in a rigid solid, with particular reference to the propagation of s thermal waves at finite speed. Nappa [18] used the theory of thermoelasticity without energy dissipation to obtain spatial energy bounds and decay estimates for the transient solutions in connection with the problem in which a thermoelastic body is deformed subject to boundary and initial data and body supplies having a compact support, provided positive definiteness assumptions are supposed upon the constitutive coefficients. Chandrasekharaiah [2] proves the uniqueness of solutions in 
the linear theory of thermoelasticity without energy dissipation, Iesan [11] establishes continuous dependence results, while Quintanilla [19] studies the question of existence. Some considerations on the propagation of plane waves in a microstretch thermoelastic diffusion solid of infinite extent can be find in the paper [12]. The Newton interpolating series proposed in [9] can be used successfully to approximate the solution of boundary value problems. The impact of initial mechanical deformation on the propagation of plane waves in a linear elastic isotropic solid crystals, is shwon in [10], while the algorithm proposed in [5] may be useful in addressing the solutions of mixed initial boundary value problem in the context of microstretch bodies. Other results regarding thermoelasticity of dipolar bodies and of microstretch bodies are presented in the papers [13]-[16].

We must emphasize that the spatial behavior of the harmonic in time vibrations has been studied by Chirita [3] in the classical linear thermoelasticity, by using a technique developed by Flavin and Knops [6] in the low frequency range. The author establishes some exponential estimates for spatial evolution of the amplitude of vibration, provided the positive definiteness of the constitutive coefficients is assumed.

The theory without energy dissipation proposed in Ciarletta [4] allows propagation of thermal waves at a finite speed.

In first part of our study we write down the mixed initial boundary value problem within context of dipolar bodies in thermoelasticity without energy dissipation. Second, we obtain some differential relations for certain crosssectional integrals. In main part we use previous relations to obtain some estimates describing how the amplitude evolves with respect to the distance to the excited base, provided the frequency of vibrations be greater than a certain critical value.

\section{Basic equations}

Let us consider that the reference configuration of a homogeneous thermoelastic dipolar body occupies a domain $B$ of three-dimensional Euclidean space and assume that $B$ is a regular and finite region with boundary $\partial B$. We denote the closure of $B$ by $\bar{B}$. We use a fixed system of rectangular Cartesian axes and adopt Cartesian tensor notation. We denote by $x_{j}$ the points of the domain $B$ and by $t \in[0, \infty)$ the temporal variable. Also, the spatial argument and the time argument of a function will be omitted when there is no likelihood of confusion. A superposed dot denotes the differentiation with respect to time $t$, and a subscript preceded by a comma denotes the diferentiation with respect to the corresponding spatial variable.

The basic system of equations of theory of anisotropic and homogeneous 
dipolar bodies in thermoelasticity without energy dissipation, consist of (see, for instance, [4])

- the equations of motion

$$
\begin{aligned}
& \left(\tau_{i j}+\sigma_{i j}\right)_{, j}+\varrho F_{i}=\varrho \ddot{u}_{i}, \\
& \mu_{i j k, i}+\sigma_{j k}+\varrho G_{j k}=I_{k r} \ddot{\varphi}_{j r} ;
\end{aligned}
$$

- the equation of energy

$$
\varrho \dot{\eta}=\frac{\varrho}{T_{0}} r-q_{i, i}
$$

- the constitutive equations

$$
\begin{aligned}
& \tau_{i j}=A_{i j m n} \varepsilon_{m n}+G_{i j m n} \gamma_{m n}+F_{m n r i j} \chi_{m n r}-D_{i j} \theta, \\
& \sigma_{i j}=G_{i j m n} \varepsilon_{m n}+B_{i j m n} \gamma_{m n}+D_{i j m n r} \chi_{m n r}-E_{i j} \theta, \\
& \mu_{i j k}=F_{i j k m n} \varepsilon_{m n}+D_{m n i j k} \gamma_{m n}+C_{i j k m n r} \chi_{m n r}-F_{i j k} \theta \\
& \varrho \eta=\frac{c}{T_{0}} \theta+D_{i j} \varepsilon_{i j}+E_{i j} \gamma_{i j}+F_{i j k} \chi_{i j k}, \\
& q_{i}=-\frac{1}{T_{0}} K_{i j} \beta_{j}
\end{aligned}
$$

- the geometric equations:

$$
2 \varepsilon_{i j}=u_{j, i}+u_{i, j}, \quad \gamma_{i j}=u_{j, i}-\varphi_{i j}, \quad \chi_{i j k}=\varphi_{i j, k} .
$$

To complete the basic equations system, we need to add the law of heat flow

$$
\dot{\beta}_{i}=\theta, i
$$

We must mention that all functions occurring in the above equations, depend on the variables $t$ and $x$. Also, all the above equations are defined for $(x, t) \in$ $\bar{B} \times[0, \infty)$.

It is important to outline that the constitutive equations (3) are derived under the additional assumption that the reference solid has a center of symmetry at each point, but is otherwise non-isotropic. In this situation, the free energy $\Psi$ has the following expression

$$
\begin{aligned}
& \varrho \Psi=\frac{1}{2} A_{i j m n} \varepsilon_{i j} \varepsilon_{m n}+G_{i j m n} \varepsilon_{i j} \gamma_{m n}+F_{m n r i j} \varepsilon_{i j} \chi_{m n r}+ \\
& +\frac{1}{2} B_{i j m n} \gamma_{i j} \gamma_{m n}+D_{i j m n r} \gamma_{i j} \chi_{m n r}+\frac{1}{2} C_{i j k m n r} \chi_{i j k} \chi_{m n r}- \\
& \quad-D_{i j} \varepsilon_{i j} \theta-E_{i j} \gamma_{i j} \theta-F_{i j} \chi_{i j k} \theta-\frac{c}{2 T_{0}} \theta^{2}+\frac{c}{2 T_{0}} K_{i j} \tau_{, i} \tau_{, j}
\end{aligned}
$$

Notations used in the above relationships have the following meanings: $\varrho$-the constant reference density, $u_{i}$-the components of displacement, $\varphi_{j k}$-the 
components of dipolar displacement, $\tau_{i j}, \sigma_{i j}, \mu_{i j k}$-the components of stress tensors, $\varepsilon_{i j}, \gamma_{i j}, \chi_{i j k}$-the components of strain tensors, $q_{i}$-the components of the heat conduction vector, $\eta$-the specific entropy, $T_{0}$-the constant reference temperature, $\theta$-the temperature variation measured from the reference temperature $T_{0}, F_{i}$-the components of body force per unit mass, $G_{j k}$-the components of dipolar body force per unit mass, $r$-the heat supply per unit mass and unit time, $I_{i j}$-the components of inertia.

The coefficients $A_{i j m n}, B_{i j m n}, \ldots, c$ are the characteristic constants of the material and they are subject to the symmetry conditions

$$
\begin{aligned}
& A_{i j m n}=A_{m n i j}=A_{i j n m}, B_{i j m n}=B_{m n i j}, G_{i j m n}=G_{j i m n}, \\
& F_{i j k m n}=F_{i j k n m}, C_{i j k m n r}=C_{m n r i j k}, E_{i j}=E_{j i}, k_{i j}=k_{j i} .
\end{aligned}
$$

Also, in (6) we denoted by $\tau$ the thermal displacement related to the temperature variation, depending on which it has the following expression

$$
\dot{\tau}=\theta
$$

If we take into consideration the constitutive equations (3) and the geometric equations (4), from the equations of motion (1) and equation of energy (2) we obtain a system of equations in terms of displacements $u_{i}$, dipolar displacements $\varphi_{i j}$ and thermal variation $\tau$, for any $(x, t) \in B \times(0, \infty)$, as

$$
\begin{gathered}
\varrho \ddot{u ̈}_{i}=\left[\left(A_{i j m n}+G_{i j m n}\right) u_{n, m}+\left(G_{m n i j}+B_{i j m n}\right)\left(u_{n, m}-\varphi_{m n}\right)+\right. \\
\left.\quad+\left(F_{m n r i j}+D_{i j m n r}\right) \varphi_{n r, m}-\left(E_{i j}+D_{i j}\right) \theta\right]_{j}+\varrho F_{i}, \\
I_{k r} \ddot{\varphi}_{j r}=\left[F_{i j k m n} u_{n, m}+D_{m n i j k}\left(u_{n, m}-\varphi_{m n}\right)+C_{i j k m n r} \varphi_{n r, m}-F_{i j k} \theta\right]_{, i}+(9) \\
\quad+G_{j k m n} u_{m, n}+B_{j k m n}\left(u_{n, m}-\varphi_{m n}\right)+D_{j k m n r} \varphi_{n r, m}-D_{j k} \theta+\varrho G_{j k}, \\
c \ddot{\tau}=-T_{0}\left[D_{i j} v_{j, i}+E_{i j}\left(v_{j, i}-\psi_{i j}\right)+F_{i j k} \psi_{j k, i}\right]+\left(K_{i j} \theta,{ }_{j}\right)_{, i}+\varrho r,
\end{gathered}
$$

\section{Preliminary results}

We consider that the dipolar thermoelastic body occupies a prismatic cylinder and denote by $D$ a cross-section in this cylinder. Also, the body is assumed be homogeneous and anisotropic. Suppose that the boundary of this section is a piecewise continuously differentiable curve, denoted by $\partial D$. Then the lateral boundary of the cylinder is $\mathcal{S}=\partial D \times[0, L]$, where $L$ is the length of the cylinder. The system of Cartesian rectangular axis has its origin in the center of the cylinder base and the positive $x_{3}$-axis is directed along the cylinder.

Assume that over the base of cylinder are given the displacements, dipolar displacements and thermal displacement, all of which are assumed harmonic in time. Also, the cylinder is assumed be free of load on the lateral boundary 
surface, that is zero body force, dipolar body force and heat supply and zero displacement, dipolar displacements and thermal displacements.

We complete the boundary value problem for the cylinder considering the following lateral boundary conditions

$$
u_{i}(x, t)=0, \varphi_{i j}(x, t)=0, \tau(x, t)=0,(x, t) \in \mathcal{S} \times(0, \infty)
$$

On the base of cylinder following boundary conditions are assumed

$$
\begin{gathered}
u_{i}\left(x_{1}, x_{2}, 0, t\right)=\tilde{u}_{i}\left(x_{1}, x_{2}\right) e^{\iota \omega t}, \varphi_{i j}\left(x_{1}, x_{2}, 0, t\right)=\tilde{\varphi}_{i j}\left(x_{1}, x_{2}\right) e^{\iota \omega t}, \\
\tau\left(x_{1}, x_{2}, 0, t\right)=\tilde{\tau}\left(x_{1}, x_{2}\right) e^{\iota \omega t},\left(x_{1}, x_{2}\right) \in D(0), t>0
\end{gathered}
$$

The functions $\tilde{u}_{i}\left(x_{1}, x_{2}\right), \tilde{\varphi}_{i j}\left(x_{1}, x_{2}\right)$ and $\tilde{\tau}\left(x_{1}, x_{2}\right)$ are given and smooth functions, $\omega$ is a given positive constant and $\iota$ is the complex unit.

We consider that the vibrations harmonic in time induced by loads defined in (11) inside the cylinder, have the form

$$
\begin{array}{r}
u_{i}\left(x_{1}, x_{2}, x_{3}, t\right)=U_{i}\left(x_{1}, x_{2}, x_{3}\right) e^{\iota \omega t}, \varphi_{i j}\left(x_{1}, x_{2}, x_{3}, t\right)=\Phi_{i j}\left(x_{1}, x_{2}, x_{3}\right) e^{\iota \omega t}, \\
\tau\left(x_{1}, x_{2}, x_{3}, t\right)=\mathcal{T}\left(x_{1}, x_{2}, x_{3}\right) e^{\iota \omega t},\left(x_{1}, x_{2}, x_{3}, t\right) \in B \times(0, \infty) .
\end{array}
$$

If we substitute $\left(u_{i}, \varphi_{i j}, \tau\right)$ from (12) in the system (9), we get that the amplitude $\left(U_{i}, \Phi_{i j}, \mathcal{T}\right)$ of the vibrations satisfies the following system of differential equations

$$
\begin{aligned}
& \varrho \omega^{2} U_{i}+\left[\left(A_{i j m n}+G_{i j m n}\right) U_{n, m}+\left(G_{m n i j}+B_{i j m n}\right)\left(U_{n, m}-\Phi_{m n}\right)+\right. \\
& \left.+\left(F_{m n r i j}+D_{i j m n r}\right) \Phi_{n r, m}-\iota \omega\left(D_{i j}+E_{i j}\right) \mathcal{T}\right]_{, j}=0 \\
& I_{k r} \omega^{2} \Phi_{j r}+\left[F_{i j k m n} U_{n, m}+D_{m n i j k}\left(U_{n, m}-\Phi_{m n}\right)+C_{i j k m n r} \Phi_{n r, m}-\iota \omega F_{i j k} T\right]_{, i}+ \\
& +G_{j k m n} U_{n, m}+B_{j k m n}\left(U_{n, m}-\Phi_{m n}\right)+D_{j k m n r} \Phi_{n r, m}-\iota \omega F_{j k} \mathcal{T}=0 \\
& \frac{c}{T_{0}} \omega^{2} \mathcal{T}+\left(\frac{1}{T_{0}} K_{i j} \mathcal{T},{ }_{j}\right)_{, i}-\iota \omega\left[D_{i j} U_{j, i}+E_{i j}\left(U_{j, i}-\Phi_{i j}\right)+F_{i j k} \Phi_{j k, i}\right]=0
\end{aligned}
$$

With the help of (12) and (10), the lateral boundary conditions become:

$$
U_{i}(x)=0, \Phi_{i j}(x)=0, \mathcal{T}(x)=0, x \in \mathcal{S}
$$

and the base boundary conditions receive the form

$$
\begin{aligned}
U_{i}\left(x_{1}, x_{2}, 0\right) & =\tilde{U}_{i}\left(x_{1}, x_{2}\right), \Phi_{i j}\left(x_{1}, x_{2}, 0\right)=\tilde{\Phi}_{i j}\left(x_{1}, x_{2}\right), \\
T\left(x_{1}, x_{2}, 0\right) & =\tilde{\mathcal{T}}\left(x_{1}, x_{2}\right),\left(x_{1}, x_{2}\right) \in D(0)
\end{aligned}
$$

If the considered cylinder has a finite length $\mathrm{L}$, then we are forced to prescribe a boundary condition on the superior base of the cylinder, that we denote by $D(L)$. 
Chirita in [3] and Ciarletta in [4] studied the spatial behavior of the amplitude for a forced oscillation, in the case of a rhombic thermoelastic materials, provided the exciting frecquency is less than a certain critical frequency.

In the following we want to estimate the evolution the amplitude with respect to the axial distance to the excited end. In this regard, we will prove first some estimates on a solution of the system of equations (13), considering the lateral boundary conditions (14) and taking into account the base boundary conditons (15).

The following theorem provides four identities, like auxiliary results on which will be based the main result.

Theorem 1. Let $\left(U_{i}, \Phi_{i j}, \mathcal{T}\right)$ be a solution of the boundary value problem consisting of Eqs. (13)-(15). Then the following equalities are satisfied

$2 \int_{D\left(x_{3}\right)}\left\{A_{i j m n} U_{j, i} \bar{U}_{n, m}+G_{i j m n}\left[U_{j, i}\left(\bar{U}_{n, m}-\bar{\Phi}_{n m}\right)+\bar{U}_{j, i}\left(U_{n, m}-\Phi_{n m}\right)\right]+\right.$

$+B_{i j m n}\left(U_{j, i}-\Phi_{i j}\right)\left(\bar{U}_{n, m}-\bar{\Phi}_{n m}\right)+F_{i j m n r}\left(U_{j, i} \bar{\Phi}_{n r, m}+\bar{U}_{j, i} \Phi_{n r, m}\right)-$

$+D_{i j m n r}\left[\left(\bar{U}_{j, i}-\bar{\Phi}_{i j}\right) \Phi_{n r, m}+\bar{\Phi}_{n r, m}\left(U_{j, i}-\Phi_{i j}\right)\right]+C_{i j k m n r} \Phi_{j k, i} \bar{\Phi}_{n r, m}+$

$-\varrho \omega^{2} U_{i} \bar{U}_{i}-I_{k r} \omega^{2} \Phi_{j r} \bar{\Phi}_{j k}+\frac{\iota}{2} \omega D_{i j}\left(\overline{\mathcal{T}} U_{j, i}-T \bar{U}_{j, i}\right)+$

$\left.+\frac{\iota}{2} \omega E_{i j}\left[\overline{\mathcal{T}}\left(U_{j, i}-\Phi_{i j}\right)-\mathcal{T}\left(\bar{U}_{j, i}-\bar{\Phi}_{i j}\right)\right]+\frac{\iota}{2} \omega F_{i j k}\left(\overline{\mathcal{T}} \Phi_{j k, i}-\mathcal{T} \bar{\Phi}_{j k, i}\right)\right\} d A=$

$=\frac{d}{d x_{3}} \int_{D\left(x_{3}\right)}\left\{\left(A_{3 j m n}+G_{3 j m n}\right)\left(U_{n, m} \bar{U}_{j}+\bar{U}_{n, m} U_{j}\right)+\iota \omega D_{3 j}\left(\overline{\mathcal{T}} U_{j}-\mathcal{T} \bar{U}_{j}\right)+\right.$

$+\left(G_{3 j m n}+B_{3 j m n}\right)\left[\left(U_{n, m}-\Phi_{n m}\right) \bar{U}_{j}+\left(\bar{U}_{n, m}-\bar{\Phi}_{n m}\right) U_{j}\right]+$

$+\left(F_{3 j m n r}+D_{3 j m n r}\right)\left(\Phi_{n r, m} \bar{U}_{j}+\bar{\Phi}_{n r, m} U_{j}\right)+\iota \omega E_{3 j}\left(\overline{\mathcal{T}} U_{j}-\mathcal{T} \bar{U}_{j}\right)+$

$+F_{3 j k m n}\left(\Phi_{m n} \bar{U}_{j, k}+\bar{\Phi}_{m n} U_{j, k}\right)+C_{3 j k m n r}\left(\Phi_{j k} \bar{\Phi}_{n r, m}+\bar{\Phi}_{j k} \Phi_{n r, m}\right)+$

$\left.+D_{3 j k m n}\left[\left(U_{k, j}-\Phi_{j k}\right) \bar{\Phi}_{m n}+\left(\bar{U}_{k, j}-\bar{\Phi}_{j k}\right) \Phi_{m n}\right]+\iota \omega F_{3 j k}\left(\overline{\mathcal{T}} \Phi_{j k}-\mathcal{T} \bar{\Phi}_{j k}\right)\right\} d A(16)$

$\int_{D\left(x_{3}\right)}\left\{\iota \omega D_{i j}\left(\overline{\mathcal{T}} U_{j, i}+\mathcal{T} \bar{U}_{j, i}\right)+\iota \omega E_{i j}\left[\overline{\mathcal{T}}\left(U_{j, i}-\Phi_{i j}\right)+\mathcal{T}\left(\bar{U}_{j, i}-\bar{\Phi}_{i j}\right)\right]+\right.$

$\left.+\iota \omega F_{i j k}\left(\overline{\mathcal{T}} \Phi_{j k, i}+\mathcal{T} \bar{\Phi}_{j k, i}\right)\right\} d A=$

$=\frac{d}{d x_{3}} \int_{D\left(x_{3}\right)}\left\{\left(A_{3 j m n}+G_{3 j m n}\right)\left(\bar{U}_{n, m} U_{j}-U_{n, m} \bar{U}_{j}\right)+\iota \omega D_{3 j}\left(\overline{\mathcal{T}} U_{j}+\mathcal{T} \bar{U}_{j}\right)+\right.$

$+\left(G_{3 j m n}+B_{3 j m n}\right)\left[\left(\bar{U}_{n, m}-\bar{\Phi}_{n m}\right) U_{j}-\left(U_{n, m}-\Phi_{n m}\right) \bar{U}_{j}\right]+$

$+\left(F_{3 j m n r}+D_{3 j m n r}\right)\left(\bar{\Phi}_{n r, m} U_{j}-\Phi_{n r, m} \bar{U}_{j}\right)+\iota \omega E_{3 j}\left(\overline{\mathcal{T}} U_{j}+\mathcal{T} \bar{U}_{j}\right)+$

$+F_{3 j k m n}\left(\bar{\Phi}_{m n} U_{j, k}-\Phi_{m n} \bar{U}_{j, k}\right)+C_{3 j k m n r}\left(\bar{\Phi}_{j k} \Phi_{n r, m}-\Phi_{j k} \bar{\Phi}_{n r, m}\right)+$

$\left.+D_{3 j k m n}\left[\left(\bar{U}_{k, j}-\bar{\Phi}_{j k}\right) \Phi_{m n}-\left(U_{k, j}-\Phi_{j k}\right) \bar{\Phi}_{m n}\right]+\iota \omega F_{3 j k}\left(\overline{\mathcal{T}} \Phi_{j k}+\mathcal{T} \bar{\Phi}_{j k}\right)\right\} d A(17)$ 


$$
\begin{aligned}
& \int_{D\left(x_{3}\right)}\left[\frac{2}{\theta_{0}}\left(K_{i j} \mathcal{T}_{, i} \overline{\mathcal{T}}_{, j}-c \omega^{2} \mathcal{T} \overline{\mathcal{T}}\right)+\iota \omega D_{i j}\left(U_{j, i} \overline{\mathcal{T}}-\bar{U}_{j, i} \mathcal{T}\right)\right] d A+ \\
& +\int_{D\left(x_{3}\right)} \iota \omega\left\{E_{i j}\left[\left(U_{j, i}-\Phi_{i j}\right) \overline{\mathcal{T}}-\left(\bar{U}_{j, i}-\bar{\Phi}_{i j}\right) \mathcal{T}\right]+F_{i j k}\left(\Phi_{j k, i} \overline{\mathcal{T}}_{-} \bar{\Phi}_{j k, i} \mathcal{T}\right)\right\} d A= \\
& =\frac{d}{d x_{3}} \int_{D\left(x_{3}\right)} \frac{1}{\theta_{0}} K_{33}\left(\overline{\mathcal{T}} \mathcal{T}_{, 3}+\mathcal{T} \overline{\mathcal{T}}_{, 3}\right) d A \\
& \int_{D\left(x_{3}\right)} \iota \omega\left\{D_{i j}\left(U_{j, i} \overline{\mathcal{T}}+U_{j, i} \mathcal{T}\right)+E_{i j}\left[\left(U_{j, i}-\Phi_{i j}\right) \overline{\mathcal{T}}+\left(\bar{U}_{j, i}-\bar{\Phi}_{i j}\right) \mathcal{T}\right]\right\} d A+ \\
& +\int_{D\left(x_{3}\right)} \iota \omega F_{i j k}\left(\Phi_{j k, i} \overline{\mathcal{T}}+\bar{\Phi}_{j k, i} \mathcal{T}\right) d A=\frac{d}{d x_{3}} \int_{D\left(x_{3}\right)} \frac{1}{\theta_{0}} K_{3 j}\left(\overline{\mathcal{T}}{ }_{, j}-\mathcal{T} \overline{\mathcal{T}},{ }_{j}\right) d A
\end{aligned}
$$

Here we denoted with $\bar{z}$ the complex conjugate of the complex number $z$.

Proof. Using equations $(13)_{1}$ and $(13)_{2}$ is easy to deduce the following equality

$$
\begin{aligned}
& \left\{\varrho \omega^{2} U_{i}+\left[\left(A_{i j m n}+G_{i j m n}\right) U_{n, m}+\left(G_{m n i j}+B_{i j m n}\right)\left(U_{n, m}-\Phi_{m n}\right)+\right.\right. \\
& \left.\left.+\left(F_{m n r i j}+D_{i j m n r}\right) \Phi_{n r, m}-\iota \omega\left(D_{i j}+E_{i j}\right) \mathcal{T}\right]_{, j}\right\} \bar{U}_{i}+ \\
& +\left\{\varrho \omega^{2} \bar{U}_{i}+\left[\left(A_{i j m n}+G_{i j m n}\right) \bar{U}_{n, m}+\left(G_{m n i j}+B_{i j m n}\right)\left(\bar{U}_{n, m}-\bar{\Phi}_{m n}\right)+\right.\right. \\
& \left.\left.+\left(F_{m n r i j}+D_{i j m n r}\right) \bar{\Phi}_{n r, m}+\iota \omega\left(D_{i j}+E_{i j}\right) \overline{\mathcal{T}}\right]_{, j}\right\} \bar{U}_{i}+ \\
& +\left\{I_{k r} \omega^{2} \Phi_{j r}+\left[F_{i j k m n} U_{n, m}+D_{m n i j k}\left(U_{n, m}-\Phi_{m n}\right)+C_{i j k m n r} \Phi_{n r, m}-\iota \omega F_{i j k} \mathcal{T}\right]_{, i}\right\} \bar{\Phi}_{j k} \\
& +\left\{G_{j k m n} U_{n, m}+B_{j k m n}\left(U_{n, m}-\Phi_{m n}\right)+D_{j k m n r} \Phi_{n r, m}-\iota \omega F_{j k} \mathcal{T}\right\} \bar{\Phi}_{j k}+ \\
& +\left\{I_{k r} \omega^{2} \bar{\Phi}_{j r}+\left[F_{i j k m n} U_{n, m}+D_{m n i j k}\left(\bar{U}_{n, m}-\bar{\Phi}_{m n}\right)+C_{i j k m n r} \bar{\Phi}_{n r, m}+\iota \omega F_{i j k} \mathcal{T}\right]_{, i}\right\} \Phi_{j k} \\
& +\left\{G_{j k m n} \bar{U}_{n, m}+B_{j k m n}\left(\bar{U}_{n, m}-\bar{\Phi}_{m n}\right)+D_{j k m n r} \bar{\Phi}_{n r, m}+\iota \omega F_{j k} \overline{\mathcal{T}}\right\} \Phi_{j k}=0 .
\end{aligned}
$$

This equality, after some obvious calculations, receives the form

$$
\begin{aligned}
& 2\left\{A_{i j m n} U_{j, i} \bar{U}_{n, m}+G_{i j m n}\left[U_{j, i}\left(\bar{U}_{n, m}-\bar{\Phi}_{n m}\right)+\bar{U}_{j, i}\left(U_{n, m}-\Phi_{n m}\right)\right]+\right. \\
& +B_{i j m n}\left(U_{j, i}-\Phi_{i j}\right)\left(\bar{U}_{n, m}-\bar{\Phi}_{n m}\right)+F_{i j m n r}\left(U_{j, i} \bar{\Phi}_{n r, m}+\bar{U}_{j, i} \Phi_{n r, m}\right)- \\
& +D_{i j m n r}\left[\left(\bar{U}_{j, i}-\bar{\Phi}_{i j}\right) \Phi_{n r, m}+\bar{\Phi}_{n r, m}\left(U_{j, i}-\Phi_{i j}\right)\right]+C_{i j k m n r} \Phi_{j k, i} \bar{\Phi}_{n r, m}+ \\
& \left.-\varrho \omega^{2} U_{i} \bar{U}_{i}-I_{k r} \omega^{2} \Phi_{j r} \bar{\Phi}_{j k}\right\}+\iota \omega D_{i j}\left(\overline{\mathcal{T}} U_{j, i}-T \bar{U}_{j, i}\right)+ \\
& +\iota \omega E_{i j}\left[\overline{\mathcal{T}}\left(U_{j, i}-\Phi_{i j}\right)-\mathcal{T}\left(\bar{U}_{j, i}-\bar{\Phi}_{i j}\right)\right]+\iota \omega F_{i j k}\left(\overline{\mathcal{T}} \Phi_{j k, i}-\mathcal{T} \bar{\Phi}_{j k, i}\right)= \\
& {\left[\left(A_{i j m n}+G_{i j m n}\right)\left(U_{n, m} \bar{U}_{j}+\bar{U}_{n, m} U_{j}\right)+\iota \omega D_{i j}\left(\overline{\mathcal{T}} U_{j}-\mathcal{T} \bar{U}_{j}\right)\right\}_{, i}+} \\
& +\left\{\left(G_{i j m n}+B_{i j m n}\right)\left[\left(U_{n, m}-\Phi_{n m}\right) \bar{U}_{j}+\left(\bar{U}_{n, m}-\bar{\Phi}_{n m}\right) U_{j}\right]\right\}_{, i}+ \\
& +\left\{\left(F_{i j m n r}+D_{i j m n r}\right)\left(\Phi_{n r, m} \bar{U}_{j}+\bar{\Phi}_{n r, m} U_{j}\right)+\iota \omega E_{i j}\left(\overline{\mathcal{T}} U_{j}-\mathcal{T} \bar{U}_{j}\right)\right\}_{, i}+
\end{aligned}
$$




$$
\begin{aligned}
& +\left\{F_{i j k m n}\left(\Phi_{m n} \bar{U}_{j, k}+\bar{\Phi}_{m n} U_{j, k}\right)+C_{i j k m n r}\left(\Phi_{j k} \bar{\Phi}_{n r, m}+\bar{\Phi}_{j k} \Phi_{n r, m}\right)\right\}_{, i}+ \\
& +\left\{D_{i j k m n}\left[\left(U_{k, j}-\Phi_{j k}\right) \bar{\Phi}_{m n}+\left(\bar{U}_{k, j}-\bar{\Phi}_{j k}\right) \Phi_{m n}\right]+\iota \omega F_{i j k}\left(\overline{\mathcal{T}} \Phi_{j k}-\mathcal{T} \bar{\Phi}_{j k}\right)\right\}_{, i}
\end{aligned}
$$

Now it is easy to obtain equality (16) by integrating equality $(21)$ over $D\left(x_{3}\right)$, then applying the divergence theorem by taking into account the lateral conditions (14).

The following equality is also obtained with the help of equations $(13)_{1}$ and $(13)_{2}$

$$
\begin{aligned}
& \left\{\varrho \omega^{2} U_{i}+\left[\left(A_{i j m n}+G_{i j m n}\right) U_{n, m}+\left(G_{m n i j}+B_{i j m n}\right)\left(U_{n, m}-\Phi_{m n}\right)+\right.\right. \\
& \left.\left.+\left(F_{m n r i j}+D_{i j m n r}\right) \Phi_{n r, m}-\iota \omega\left(D_{i j}+E_{i j}\right) \mathcal{T}\right]_{, j}\right\} \bar{U}_{i}+ \\
& -\left\{\varrho \omega^{2} \bar{U}_{i}+\left[\left(A_{i j m n}+G_{i j m n}\right) \bar{U}_{n, m}+\left(G_{m n i j}+B_{i j m n}\right)\left(\bar{U}_{n, m}-\bar{\Phi}_{m n}\right)+\right.\right. \\
& \left.\left.-\left(F_{m n r i j}+D_{i j m n r}\right) \bar{\Phi}_{n r, m}+\iota \omega\left(D_{i j}+E_{i j}\right) \overline{\mathcal{T}}\right]_{, j}\right\} \bar{U}_{i}+ \\
& +\left\{I_{k r} \omega^{2} \Phi_{j r}+\left[F_{i j k m n} U_{n, m}+D_{m n i j k}\left(U_{n, m}-\Phi_{m n}\right)+C_{i j k m n r} \Phi_{n r, m}-\iota \omega F_{i j k} \mathcal{T}\right]_{, i}\right\} \bar{\Phi}_{j k} \\
& +\left\{G_{j k m n} U_{n, m}+B_{j k m n}\left(U_{n, m}-\Phi_{m n}\right)+D_{j k m n r} \Phi_{n r, m}-\iota \omega F_{j k} \mathcal{T}\right\} \bar{\Phi}_{j k}+ \\
& -\left\{I_{k r} \omega^{2} \bar{\Phi}_{j r}+\left[F_{i j k m n} U_{n, m}+D_{m n i j k}\left(\bar{U}_{n, m}-\bar{\Phi}_{m n n}\right)+C_{i j k m n r} \bar{\Phi}_{n r, m}+\iota \omega F_{i j k} \mathcal{T}{ }_{, i}\right\} \Phi_{j k}\right. \\
& -\left\{G_{j k m n} \bar{U}_{n, m}+B_{j k m n}\left(\bar{U}_{n, m}-\bar{\Phi}_{m n}\right)+D_{j k m n r} \bar{\Phi}_{n r, m}+\iota \omega F_{j k} \mathcal{\mathcal { T }}\right\} \Phi_{j k}=0 .
\end{aligned}
$$

After some simple calculations, we can rewrite equality (22) in the form

$$
\begin{aligned}
& \iota \omega D_{i j}\left(\overline{\mathcal{T}} U_{j, i}+\mathcal{T} \bar{U}_{j, i}\right)+\iota \omega E_{i j}\left[\overline{\mathcal{T}}\left(U_{j, i}-\Phi_{i j}\right)+\mathcal{T}\left(\bar{U}_{j, i}-\bar{\Phi}_{i j}\right)\right]+ \\
& +\iota \omega F_{i j k}\left(\overline{\mathcal{T}} \Phi_{j k, i}+\mathcal{T} \bar{\Phi}_{j k, i}\right)= \\
& =\left[\left(A_{i j m n}+G_{i j m n}\right)\left(\bar{U}_{n, m} U_{j}-U_{n, m} \bar{U}_{j}\right)+\iota \omega D_{i j}\left(\overline{\mathcal{T}} U_{j}+\mathcal{T} \bar{U}_{j}\right)\right]_{, i}+ \\
& \left.+\left\{\left(G_{i j m n}+B_{i j m n}\right)\left[\left(\bar{U}_{n, m}-\bar{\Phi}_{n m}\right) U_{j}-\left(U_{n, m}-\Phi_{n m}\right) \bar{U}_{j}\right]\right\}_{, i}+{ }{ }_{i j}\right) \\
& +\left[\left(F_{i j m n r}+D_{i j m n r}\right)\left(\bar{\Phi}_{n r, m} U_{j}-\Phi_{n r, m} \bar{U}_{j}\right)+\iota \omega E_{i j}\left(\overline{\mathcal{T}} U_{j}+\mathcal{T} \bar{U}_{j}\right)\right]_{, i}+ \\
& +\left[F_{i j k m n}\left(\bar{\Phi}_{m n} U_{j, k}-\Phi_{m n} \bar{U}_{j, k}\right)+C_{i j k m n r}\left(\bar{\Phi}_{j k} \Phi_{n r, m}-\Phi_{j k} \bar{\Phi}_{n r, m}\right)\right], i \\
& +\left[D_{i j k m n}\left[\left(\bar{U}_{k, j}-\bar{\Phi}_{j k}\right) \Phi_{m n}-\left(U_{k, j}-\Phi_{j k}\right) \bar{\Phi}_{m n}\right]+\iota \omega F_{i j k}\left(\overline{\mathcal{T}} \Phi_{j k}+\mathcal{T} \bar{\Phi}_{j k}\right)\right]_{, i}
\end{aligned}
$$

If we integrate equality (23) over $D\left(x_{3}\right)$, then apply the divergence theorem and take into account the lateral conditions (14) we get the equality (17). Now we use the equation $(13)_{3}$, in order to deduce the equality

$$
\begin{aligned}
& \overline{\mathcal{T}}\left\{\frac{c}{T_{0}} \omega^{2} \mathcal{T}+\left(\frac{1}{T_{0}} K_{i j} \mathcal{T}, j\right)_{, i}-\iota \omega\left[D_{i j} U_{j, i}+E_{i j}\left(U_{j, i}-\Phi_{i j}\right)+F_{i j k} \Phi_{j k, i}\right]\right\}+ \\
& +\mathcal{T}\left\{\frac{c}{T_{0}} \omega^{2} \overline{\mathcal{T}}+\left(\frac{1}{T_{0}} K_{i j} T,{ }_{j}\right)_{, i}-\iota \omega\left[D_{i j} \bar{U}_{j, i}+E_{i j}\left(\bar{U}_{j, i}-\bar{\Phi}_{i j}\right)+F_{i j k} \bar{\Phi}_{j k, i}\right]\right\}
\end{aligned}
$$


It is easy to see that equality (24) can be written in the form

$$
\begin{gathered}
\frac{2}{T_{0}}\left(K_{i j} \mathcal{T},{ }_{i} \overline{\mathcal{T}},{ }_{j}-c \omega^{2} \mathcal{T} \overline{\mathcal{T}}\right)+\iota \omega D_{i j}\left(U_{j, i} \overline{\mathcal{T}}-\bar{U}_{j, i} \mathcal{T}\right)+ \\
+\iota \omega\left\{E_{i j}\left[\left(U_{j, i}-\Phi_{i j}\right) \overline{\mathcal{T}}-\left(\bar{U}_{j, i}-\bar{\Phi}_{i j}\right) \mathcal{T}\right]+F_{i j k}\left(\Phi_{j k, i} \overline{\mathcal{T}}-\bar{\Phi}_{j k, i} \mathcal{T}\right)\right\}= \\
=\frac{1}{\theta_{0}}\left[K_{i j}\left(\overline{\mathcal{T}} \mathcal{T},{ }_{j}+\mathcal{T} \overline{\mathcal{T}}_{, j}\right)\right], i
\end{gathered}
$$

If we integrate equality $(25)$ over $D\left(x_{3}\right)$, then apply the divergence theorem and take into account the lateral conditions (14) we get the equality (18).

*** Finally, we use again equation $(13)_{3}$ based on which we deduce equality

$\overline{\mathcal{T}}\left[\left(\frac{1}{\theta_{0}} K_{i j} \mathcal{T}_{, j}\right)_{, i}-\iota \omega D_{i j} U_{j, i}-\iota \omega E_{i j}\left(U_{j, i}-\Phi_{i j}\right)-\iota \omega F_{i j k} \Phi_{j k, i}+\frac{c}{\theta_{0}} \omega^{2} \mathcal{T}\right]-$

$-\mathcal{T}\left[\left(\frac{1}{\theta_{0}} K_{i j} \overline{\mathcal{T}}_{, j}\right)_{, i}+\iota \omega D_{i j} \bar{U}_{j, i}+\iota \omega E_{i j}\left(\bar{U}_{j, i}-\bar{\Phi}_{i j}\right)+\iota \omega F_{i j k} \bar{\Phi}_{j k, i}-\frac{c}{\theta_{0}} \omega^{2} \overline{\mathcal{T}}\right]=0(26)$

Of course, this equality can be rewritten as follows

$$
\begin{array}{r}
\iota \omega D_{i j}\left(U_{j, i} \overline{\mathcal{T}}+\bar{U}_{j, i} T\right)+\iota \omega E_{i j}\left[\left(\bar{U}_{j, i}-\bar{\Phi}_{i j}\right) \mathcal{T}+\left(U_{j, i}-\Phi_{i j}\right) \overline{\mathcal{T}}\right]+ \\
+\iota \omega F_{i j k}\left(\bar{\Phi}_{j k, i} \mathcal{T}+\Phi_{j k, i} \overline{\mathcal{T}}\right)=\left[\frac{1}{\theta_{0}} K_{i j}\left(\overline{\mathcal{T}} \mathcal{T},{ }_{j}-\mathcal{T} \overline{\mathcal{T}}_{, j}\right)\right]_{, i}
\end{array}
$$

Integrate equality $(27)$ over $D\left(x_{3}\right)$, apply the divergence theorem and if we use the lateral conditions (14) we get the equality (19) the proof of Theorem 1 is completed.

Now we demonstrate two other identities that are useful to prove the main result of our study.

Theorem 2. If $\left(U_{i}, \Phi_{i j}, \mathcal{T}\right)$ is a solution of the boundary value problem consisting of Eqs. (13)-(15), then take place these identities

$$
\begin{aligned}
& \int_{D\left(x_{3}\right)}\left\{A_{i j m n} U_{j, i} \bar{U}_{n, m}+G_{i j m n}\left[U_{j, i}\left(\bar{U}_{n, m}-\bar{\Phi}_{n m}\right)+\bar{U}_{j, i}\left(U_{n, m}-\Phi_{n m}\right)\right]+\right. \\
& +B_{i j m n}\left(U_{j, i}-\Phi_{i j}\right)\left(\bar{U}_{n, m}-\bar{\Phi}_{n m}\right)+F_{i j m n r}\left(U_{j, i} \bar{\Phi}_{n r, m}+\bar{U}_{j, i} \Phi_{n r, m}\right)- \\
& +D_{i j m n r}\left[\left(\bar{U}_{j, i}-\bar{\Phi}_{i j}\right) \Phi_{n r, m}+\bar{\Phi}_{n r, m}\left(U_{j, i}-\Phi_{i j}\right)\right]+C_{i j k m n r} \Phi_{j k, i} \bar{\Phi}_{n r, m}+ \\
& -3 \omega^{2}\left(\varrho U_{i} \bar{U}_{i}+I_{k r} \Phi_{j r} \bar{\Phi}_{j k}\right)-\iota \omega D_{i j}\left(\overline{\mathcal{T}} U_{j, i}-\mathcal{T} \bar{U}_{j, i}\right)- \\
& \left.-\iota \omega E_{i j}\left[\overline{\mathcal{T}}\left(U_{j, i}-\Phi_{i j}\right)-\mathcal{T}\left(\bar{U}_{j, i}-\bar{\Phi}_{i j}\right)\right]-\iota \omega F_{i j k}\left(\overline{\mathcal{T}} \Phi_{j k, i}-\mathcal{T} \bar{\Phi}_{j k, i}\right)\right\} d A- \\
& -\iota \omega \int_{D\left(x_{3}\right)}\left\{x_{p} D_{i j}\left(\overline{\mathcal{T}}_{, p} U_{j, i}-\mathcal{T},{ }_{, p} \bar{U}_{j, i}\right)+x_{p} F_{i j k}\left(\overline{\mathcal{T}}, p \Phi_{j k, i}-\mathcal{T}, p \bar{\Phi}_{j k, i}\right)+\right. \\
& \left.+x_{p} E_{i j}\left[\overline{\mathcal{T}}_{, p}\left(U_{j, i}-\Phi_{i j}\right)-\mathcal{T}, p\left(\bar{U}_{j, i}-\bar{\Phi}_{i j}\right)\right]\right\} d A=
\end{aligned}
$$




$$
\begin{aligned}
& =-\frac{d}{d x_{3}} \int_{D\left(x_{3}\right)}\left\{\left[A_{3 j m n} U_{n, m}+G_{3 j m n}\left(U_{n, m}-\Phi_{n m}\right)+F_{3 j m n r} \Phi_{n r, m}-\iota \omega D_{3 j} \mathcal{T}\right] x_{p} \bar{U}_{j, p}\right. \\
& \left.-\left[A_{3 j m n} \bar{U}_{n, m}+G_{3 j m n}\left(\bar{U}_{n, m}-\bar{\Phi}_{n m}\right)+F_{3 j m n r} \bar{\Phi}_{n r, m}+\iota \omega D_{3 j} \overline{\mathcal{T}}\right] x_{p} U_{j, p}\right\} d A \\
& -\frac{d}{d x_{3}} \int_{D\left(x_{3}\right)}\left\{\left[G_{3 j m n} U_{n, m}+B_{3 j m n}\left(U_{n, m}-\Phi_{n m}\right)+D_{3 j m n r} \Phi_{n r, m}-\iota \omega E_{3 j} \mathcal{T}\right] x_{p} \bar{U}_{j, p}\right. \\
& \left.-\left[G_{3 j m n} \bar{U}_{n, m}+B_{3 j m n}\left(\bar{U}_{n, m}-\bar{\Phi}_{n m}\right)+D_{3 j m n r} \bar{\Phi}_{n r, m}+\iota \omega E_{3 j} \overline{\mathcal{T}}\right] x_{p} U_{j, p}\right\} d A \\
& -\frac{d}{d x_{3}} \int_{D\left(x_{3}\right)}\left\{\left[F_{3 j k m n} U_{n, m}+D_{3 j k m n}\left(U_{n, m}-\Phi_{n m}\right)+C_{3 j k m n r} \Phi_{n r, m}-\iota \omega F_{3 j k} \mathcal{T}\right] x_{p} \bar{\Phi}_{j k, p}\right. \\
& \left.-\left[F_{3 j k m n} \bar{U}_{n, m}+D_{3 j k m n}\left(\bar{U}_{n, m}-\bar{\Phi}_{n m}\right)+C_{3 j k m n r} \bar{\Phi}_{n r, m}+\iota \omega F_{3 j k} \overline{\mathcal{T}}\right] x_{p} \Phi_{j k, p}\right\} d A \\
& +\frac{d}{d x_{3}} \int_{D\left(x_{3}\right)} x_{3}\left\{A_{i j m n} U_{j, i} \bar{U}_{n, m}+G_{i j m n}\left[U_{j, i}\left(\bar{U}_{n, m}-\bar{\Phi}_{n m}\right)+\bar{U}_{j, i}\left(U_{n, m}-\Phi_{n m}\right)\right]\right. \\
& +B_{i j m n}\left(U_{j, i}-\Phi_{i j}\right)\left(\bar{U}_{n, m}-\bar{\Phi}_{n m}\right)+F_{i j m n r}\left(U_{j, i} \bar{\Phi}_{n r, m}+\bar{U}_{j, i} \Phi_{n r, m}\right)- \\
& \left.+D_{i j m n r}\left[\left(\bar{U}_{j, i}-\bar{\Phi}_{i j}\right) \Phi_{n r, m}+\bar{\Phi}_{n r, m}\left(U_{j, i}-\Phi_{i j}\right)\right]+C_{i j k m n r} \Phi_{j k, i} \bar{\Phi}_{n r, m}\right\} d A+ \\
& -\frac{d}{d x_{3}} \int_{D\left(x_{3}\right)} x_{3}\left[\omega^{2}\left(\varrho U_{i} \bar{U}_{i}+I_{k r} \Phi_{j r} \bar{\Phi}_{j k}\right)+\iota \omega D_{i j}\left(\overline{\mathcal{T}} U_{j, i}-\mathcal{T} \bar{U}_{j, i}\right)\right] d A- \\
& -\iota \omega \frac{d}{d x_{3}} \int_{D\left(x_{3}\right)} x_{3}\left\{E_{i j}\left[\overline{\mathcal{T}}\left(U_{j, i}-\Phi_{i j}\right)-\mathcal{T}\left(\bar{U}_{j, i}-\bar{\Phi}_{i j}\right)\right]+F_{i j k}\left(\overline{\mathcal{T}} \Phi_{j k, i}-\mathcal{T} \bar{\Phi}_{j k, i}\right)\right\} d A \\
& +\int_{\partial D\left(x_{3}\right)} x_{p} n_{p}\left(A_{i \alpha m \beta} \frac{\partial U_{i}}{\partial n} \frac{\partial \bar{U}_{m}}{\partial n}+F_{i \alpha k m \beta} \frac{\partial U_{i}}{\partial n} \frac{\partial \bar{\Phi}_{k m}}{\partial n}+C_{i j \alpha k m \beta} \frac{\partial \Phi_{i j}}{\partial n} \frac{\partial \bar{\Phi}_{k m}}{\partial n}\right) n_{\alpha} n_{\beta} d s \\
& \int_{D\left(x_{3}\right)}\left[\frac{1}{T_{0}}\left(K_{i j} \mathcal{T},{ }_{i} \overline{\mathcal{T}}_{, j}-3 c \omega^{2} \mathcal{T} \overline{\mathcal{T}}\right)+\iota \omega D_{i j} x_{p}\left(\bar{U}_{j, i} \mathcal{T}, p-U_{j, i} \overline{\mathcal{T}}, p\right)\right] d A \\
& +\int_{D\left(x_{3}\right)} \iota \omega E_{i j} x_{p}\left\{\left[\left(\bar{U}_{j, i}-\bar{\Phi}_{i j}\right) \mathcal{T}_{, p}-\left(U_{j, i}-\Phi_{i j}\right) \overline{\mathcal{T}}_{, p}\right]+D_{i j}\left(\bar{U}_{j, i} \mathcal{T}_{, p}-U_{j, i} \overline{\mathcal{T}}_{, p}\right)\right\} d A \\
& +\int_{D\left(x_{3}\right)} \iota \omega x_{p} F_{i j k}\left(\bar{\Phi}_{j k, i} \mathcal{T}_{, p}-\Phi_{j k, i} \overline{\mathcal{T}}_{, p}\right) d A+\int_{\partial D\left(x_{3}\right)} \frac{1}{T_{0}} x_{p} n_{p} K_{\alpha \beta} n_{\alpha} n_{\beta} \frac{\partial \mathcal{T}}{\partial n} \frac{\partial \overline{\mathcal{T}}}{\partial n} d s \\
& =-\frac{d}{d x_{3}} \int_{D\left(x_{3}\right)} \frac{1}{T_{0}}\left[x_{\alpha} K_{3 \beta}\left(\overline{\mathcal{T}}_{, \alpha} \mathcal{T}_{, \beta}+\mathcal{T}_{, \alpha} \overline{\mathcal{T}}_{, \beta}\right)+x_{\alpha} K_{33}\left(\mathcal{T}_{, 3} \overline{\mathcal{T}}_{, \alpha}+\overline{\mathcal{T}}_{3} \mathcal{T}_{, \alpha}\right)\right] d A \\
& -\frac{d}{d x_{3}} \int_{D\left(x_{3}\right)} \frac{x_{3}}{T_{0}}\left(K_{33} \mathcal{T}_{, 3} \overline{\mathcal{T}}_{3}-K_{\alpha \beta} \mathcal{T}, \alpha \overline{\mathcal{T}}, \beta+c \omega^{2} \mathcal{T} \overline{\mathcal{T}}\right) d A
\end{aligned}
$$

Proof. Multiplying equation $(13)_{1}$ with $x_{p} \bar{U}_{i, p}$ and equation $(13)_{2}$ with $x_{p} \bar{\Phi}_{j k, p}$, then we add the resulting relations. We then made a similar combination regarding to the conjugates of equations $(13)_{1}$ and $(13)_{2}$. So, we 
obtain

$$
\begin{aligned}
& \left\{\left[\left(A_{i j m n}+G_{i j m n}\right) U_{n, m}+\left(G_{m n i j}+B_{i j m n}\right)\left(U_{n, m}-\Phi_{m n}\right)+\right.\right. \\
& \left.\left.+\left(F_{m n r i j}+D_{i j m n r}\right) \Phi_{n r, m}-\iota \omega\left(D_{i j}+E_{i j}\right) \mathcal{T}\right]_{, j}+\varrho \omega^{2} U_{i}\right\} x_{p} \bar{U}_{i, p}+ \\
& +\left\{\left[F_{i j k m n} U_{n, m}+D_{m n i j k}\left(U_{n, m}-\Phi_{m n}\right)+C_{i j k m n r} \Phi_{n r, m}-\iota \omega F_{i j k} \mathcal{T}\right], i\right. \\
& \left.+G_{j k m n} U_{n, m}+B_{j k m n}\left(U_{n, m}-\Phi_{m n}\right)+D_{j k m n r} \Phi_{n r, m}-\iota \omega F_{j k} \mathcal{T}+I_{k r} \omega^{2} \Phi_{j r}\right\} x_{p} \bar{\Phi}_{j k, p} \\
& +\left\{\left[\left(A_{i j m n}+G_{i j m n}\right) \bar{U}_{n, m}+\left(G_{m n i j}+B_{i j m n}\right)\left(\bar{U}_{n, m}-\bar{\Phi}_{m n}\right)+\right.\right. \\
& \left.+\left(F_{m n r i j}+D_{i j m n r}\right) \bar{\Phi}_{n r, m}+\iota \omega\left(D_{i j}+E_{i j}\right) \overline{\mathcal{T}}\right], j \\
& \left.+\varrho \omega^{2} \bar{U}_{i}\right\} x_{p} U_{i, p}+ \\
& +\left\{\left[F_{i j k m n} \bar{U}_{n, m}+D_{m n i j k}\left(\bar{U}_{n, m}-\bar{\Phi}_{m n}\right)+C_{i j k m n r} \bar{\Phi}_{n r, m}+\iota \omega F_{i j k} \overline{\mathcal{T}}\right], i\right. \\
& \left.+G_{j k m n}+\bar{U}_{n, m}+B_{j k m n}\left(\bar{U}_{n, m}-\bar{\Phi}_{m n}\right)+D_{j k m n r} \bar{\Phi}_{n r, m}+\iota \omega F_{j k} \overline{\mathcal{T}}+I_{k r} \omega^{2} \bar{\Phi}_{j r}\right\} x_{p} \Phi_{j k, p}=0
\end{aligned}
$$

Using simple rule of derivation of the product, we can rewrite the last equality in the following form

$$
\begin{aligned}
& \left\{\left[\left(A_{i j m n}+G_{i j m n}\right) U_{n, m}+\left(G_{m n i j}+B_{i j m n}\right)\left(U_{n, m}-\Phi_{m n}\right)+\right.\right. \\
& \left.\left.+\left(F_{m n r i j}+D_{i j m n r}\right) \Phi_{n r, m}-\iota \omega\left(D_{i j}+E_{i j}\right) \mathcal{T}\right] x_{p} \bar{U}_{i, p}\right\}_{, j}+\varrho \omega^{2} x_{p} U_{i} \bar{U}_{i, p}- \\
& -\left[\left(A_{i j m n}+G_{i j m n}\right) U_{n, m}+\left(G_{m n i j}+B_{i j m n}\right)\left(U_{n, m}-\Phi_{m n}\right)+\right. \\
& \left.+\left(F_{m n r i j}+D_{i j m n r}\right) \Phi_{n r, m}-\iota \omega\left(D_{i j}+E_{i j}\right) \mathcal{T}\right] x_{p} \bar{U}_{i, p j}+ \\
& +\left\{\left[F_{i j k m n} U_{n, m}+D_{m n i j k}\left(U_{n, m}-\Phi_{m n}\right)+C_{i j k m n r} \Phi_{n r, m}-\iota \omega F_{i j k} \mathcal{T}\right] x_{p} \bar{\Phi}_{j k, p}\right\}_{, i}+ \\
& +\left[G_{j k m n} U_{n, m}+B_{j k m n}\left(U_{n, m}-\Phi_{m n}\right)+D_{j k m n r} \Phi_{n r, m}-\iota \omega F_{j k} \mathcal{T}\right] x_{p} \bar{\Phi}_{j k, p}+ \\
& +I_{k r} \omega^{2} x_{p} \Phi_{j r} \bar{\Phi}_{j k, p}+I_{k r} \omega^{2} x_{p} \bar{\Phi}_{j r} \Phi_{j k, p}- \\
& -\left[F_{i j k m n} U_{n, m}+D_{m n i j k}\left(U_{n, m}-\Phi_{m n}\right)+C_{i j k m n r} \Phi_{n r, m}-\iota \omega F_{i j k} \mathcal{T}\right] x_{p} \bar{\Phi}_{j k, p i}+(31) \\
& +\left\{\left[\left(A_{i j m n}+G_{i j m n}\right) \bar{U}_{n, m}+\left(G_{m n i j}+B_{i j m n}\right)\left(\bar{U}_{n, m}-\bar{\Phi}_{m n}\right)+\right.\right. \\
& \left.\left.+\left(F_{m n r i j}+D_{i j m n r}\right) \bar{\Phi}_{n r, m}-\iota \omega\left(D_{i j}+E_{i j}\right) \overline{\mathcal{T}}\right] x_{p} U_{i, p}\right\}_{, j}+\varrho \omega^{2} x_{p} \bar{U}_{i} U_{i, p}- \\
& -\left[\left(A_{i j m n}+G_{i j m n}\right) \bar{U}_{n, m}+\left(G_{m n i j}+B_{i j m n}\right)\left(\bar{U}_{n, m}-\bar{\Phi}_{m n}\right)+\right. \\
& \left.+\left(F_{m n r i j}+D_{i j m n r}\right) \bar{\Phi}_{n r, m}-\iota \omega\left(D_{i j}+E_{i j}\right) \overline{\mathcal{T}}\right] x_{p} U_{i, p j}+ \\
& +\left\{\left[F_{i j k m n} \bar{U}_{n, m}+D_{m n i j k}\left(\bar{U}_{n, m}-\bar{\Phi}_{m n}\right)+C_{i j k m n r} \bar{\Phi}_{n r, m}-\iota \omega F_{i j k} \overline{\mathcal{T}}\right] x_{p} \Phi_{j k, p}\right\}_{, i}+ \\
& +\left[G_{j k m n} \bar{U}_{n, m}+B_{j k m n}\left(\bar{U}_{n, m}-\bar{\Phi}_{m n}\right)+D_{j k m n r} \bar{\Phi}_{n r, m}-\iota \omega F_{j k} \overline{\mathcal{T}}\right] x_{p} \Phi_{j k, p}+ \\
& -\left[F_{i j k m n} \bar{U}_{n, m}+D_{m n i j k}\left(\bar{U}_{n, m}-\bar{\Phi}_{m n}\right)+C_{i j k m n r} \bar{\Phi}_{n r, m}-\iota \omega F_{i j k} \overline{\mathcal{T}}\right] x_{p} \Phi_{j k, p i}
\end{aligned}
$$


An equivalent form to (32), easily to obtain, is the following

$$
\begin{aligned}
& A_{i j m n} U_{j, i} \bar{U}_{n, m}+G_{i j m n}\left[U_{j, i}\left(\bar{U}_{n, m}-\bar{\Phi}_{n m}\right)+\bar{U}_{j, i}\left(U_{n, m}-\Phi_{n m}\right)\right]+ \\
& +B_{i j m n}\left(U_{j, i}-\Phi_{i j}\right)\left(\bar{U}_{n, m}-\bar{\Phi}_{n m}\right)+F_{i j m n r}\left(U_{j, i} \bar{\Phi}_{n r, m}+\bar{U}_{j, i} \Phi_{n r, m}\right)- \\
& +D_{i j m n r}\left[\left(\bar{U}_{j, i}-\bar{\Phi}_{i j}\right) \Phi_{n r, m}+\bar{\Phi}_{n r, m}\left(U_{j, i}-\Phi_{i j}\right)\right]+C_{i j k m n r} \Phi_{j k, i} \bar{\Phi}_{n r, m}+ \\
& -3 \omega^{2}\left(\varrho U_{i} \bar{U}_{i}+I_{k r} \Phi_{j r} \bar{\Phi}_{j k}\right)-\iota \omega D_{i j}\left(\overline{\mathcal{T}} U_{j, i}-\mathcal{T} \bar{U}_{j, i}\right)- \\
& -\iota \omega E_{i j}\left[\overline{\mathcal{T}}\left(U_{j, i}-\Phi_{i j}\right)-\mathcal{T}\left(\bar{U}_{j, i}-\bar{\Phi}_{i j}\right)\right]-\iota \omega F_{i j k}\left(\overline{\mathcal{T}} \Phi_{j k, i}-\mathcal{T} \bar{\Phi}_{j k, i}\right)- \\
& -\iota \omega\left\{x_{p} D_{i j}\left(\overline{\mathcal{T}}_{, p} U_{j, i}-\mathcal{T},{ }_{p} \bar{U}_{j, i}\right)+x_{p} F_{i j k}\left(\overline{\mathcal{T}}_{, p} \Phi_{j k, i}-\mathcal{T},{ }_{p} \bar{\Phi}_{j k, i}\right)+\right. \\
& \left.+x_{p} E_{i j}\left[\overline{\mathcal{T}}_{, p}\left(U_{j, i}-\Phi_{i j}\right)-\mathcal{T}_{, p}\left(\bar{U}_{j, i}-\bar{\Phi}_{i j}\right)\right]\right\}= \\
& =-\left\{\left[A_{i j m n} U_{n, m}+G_{i j m n}\left(U_{n, m}-\Phi_{n m}\right)+F_{i j m n r} \Phi_{n r, m}-\iota \omega D_{i j} \mathcal{T}\right] x_{p} \bar{U}_{i, p}\right\}_{, j} \\
& -\left\{\left[A_{i j m n} \bar{U}_{n, m}+G_{i j m n}\left(\bar{U}_{n, m}-\bar{\Phi}_{n m}\right)+F_{i j m n r} \bar{\Phi}_{n r, m}+\iota \omega D_{i j} \overline{\mathcal{T}}\right] x_{p} U_{i, p}\right\}_{, j} \\
& -\left\{\left[G_{i j m n} U_{n, m}+B_{i j m n}\left(U_{n, m}-\Phi_{n m}\right)+D_{i j m n r} \Phi_{n r, m}-\iota \omega E_{i j} \mathcal{T}\right] x_{p} \bar{U}_{i, p}\right\}_{, j} \\
& -\left\{\left[G_{i j m n} \bar{U}_{n, m}+B_{i j m n}\left(\bar{U}_{n, m}-\bar{\Phi}_{n m}\right)+D_{i j m n r} \bar{\Phi}_{n r, m}+\iota \omega E_{i j} \overline{\mathcal{T}}\right] x_{p} U_{i, p}\right\}_{, j} \\
& -\left\{\left[F_{i j k m n} U_{n, m}+D_{i j k m n}\left(U_{n, m}-\Phi_{n m}\right)+C_{i j k m n r} \Phi_{n r, m}-\iota \omega F_{3 j k} \mathcal{T}\right] x_{p} \bar{\Phi}_{i k, p}\right\}_{, j} \\
& -\left\{\left[F_{i j k m n} \bar{U}_{n, m}+D_{i j k m n}\left(\bar{U}_{n, m}-\bar{\Phi}_{n m}\right)+C_{i j k m n r} \bar{\Phi}_{n r, m}+\iota \omega F_{i j k} \overline{\mathcal{T}}\right] x_{p} \Phi_{i k, p}\right\}_{, j} \\
& +\left\{x_{p} A_{i j m n} U_{j, i} \bar{U}_{n, m}+x_{p} G_{i j m n}\left[U_{j, i}\left(\bar{U}_{n, m}-\bar{\Phi}_{n m}\right)+\bar{U}_{j, i}\left(U_{n, m}-\Phi_{n m}\right)\right]\right. \\
& +x_{p} B_{i j m n}\left(U_{j, i}-\Phi_{i j}\right)\left(\bar{U}_{n, m}-\bar{\Phi}_{n m}\right)+x_{p} F_{i j m n r}\left(U_{j, i} \bar{\Phi}_{n r, m}+\bar{U}_{j, i} \Phi_{n r, m}\right)- \\
& \left.+x_{p} D_{i j m n r}\left[\left(\bar{U}_{j, i}-\bar{\Phi}_{i j}\right) \Phi_{n r, m}+\bar{\Phi}_{n r, m}\left(U_{j, i}-\Phi_{i j}\right)\right]+x_{p} C_{i j k m n r} \Phi_{j k, i} \bar{\Phi}_{n r, m}\right\}_{, p} \\
& -\left\{x_{p}\left[\omega^{2}\left(\varrho U_{i} \bar{U}_{i}+I_{k r} \Phi_{j r} \bar{\Phi}_{j k}\right)+\iota \omega D_{i j}\left(\overline{\mathcal{T}} U_{j, i}-\mathcal{T} \bar{U}_{j, i}\right)\right]\right\}_{, p}- \\
& -\iota \omega\left\{x_{p} E_{i j}\left[\overline{\mathcal{T}}\left(U_{j, i}-\Phi_{i j}\right)-\mathcal{T}\left(\bar{U}_{j, i}-\bar{\Phi}_{i j}\right)\right]+x_{p} F_{i j k}\left(\overline{\mathcal{T}} \Phi_{j k, i}-\mathcal{T} \bar{\Phi}_{j k, i}\right)\right\}_{, p}
\end{aligned}
$$

Equality (32) can be integrated on $D\left(x_{3}\right)$ and if we take into account the lateral boundary condition (14) we will be lead to the equality

$$
\begin{aligned}
& \int_{D\left(x_{3}\right)}\left\{A_{i j m n} U_{j, i} \bar{U}_{n, m}+G_{i j m n}\left[U_{j, i}\left(\bar{U}_{n, m}-\bar{\Phi}_{n m}\right)+\bar{U}_{j, i}\left(U_{n, m}-\Phi_{n m}\right)\right]+\right. \\
& +B_{i j m n}\left(U_{j, i}-\Phi_{i j}\right)\left(\bar{U}_{n, m}-\bar{\Phi}_{n m}\right)+F_{i j m n r}\left(U_{j, i} \bar{\Phi}_{n r, m}+\bar{U}_{j, i} \Phi_{n r, m}\right)- \\
& +D_{i j m n r}\left[\left(\bar{U}_{j, i}-\bar{\Phi}_{i j}\right) \Phi_{n r, m}+\bar{\Phi}_{n r, m}\left(U_{j, i}-\Phi_{i j}\right)\right]+C_{i j k m n r} \Phi_{j k, i} \bar{\Phi}_{n r, m}+
\end{aligned}
$$




$$
\begin{aligned}
& -3 \omega^{2}\left(\varrho U_{i} \bar{U}_{i}+I_{k r} \Phi_{j r} \bar{\Phi}_{j k}\right)-\iota \omega D_{i j}\left(\overline{\mathcal{T}} U_{j, i}-\mathcal{T} \bar{U}_{j, i}\right)- \\
& \left.-\iota \omega E_{i j}\left[\overline{\mathcal{T}}\left(U_{j, i}-\Phi_{i j}\right)-\mathcal{T}\left(\bar{U}_{j, i}-\bar{\Phi}_{i j}\right)\right]-\iota \omega F_{i j k}\left(\overline{\mathcal{T}} \Phi_{j k, i}-\mathcal{T} \bar{\Phi}_{j k, i}\right)\right\} d A- \\
& -\iota \omega \int_{D\left(x_{3}\right)}\left\{x_{p} D_{i j}\left(\overline{\mathcal{T}}_{, p} U_{j, i}-\mathcal{T}_{, p} \bar{U}_{j, i}\right)+x_{p} F_{i j k}\left(\overline{\mathcal{T}}_{, p} \Phi_{j k, i}-\mathcal{T}_{, p} \bar{\Phi}_{j k, i}\right)+\right. \\
& \left.+x_{p} E_{i j}\left[\overline{\mathcal{T}}_{, p}\left(U_{j, i}-\Phi_{i j}\right)-\mathcal{T}_{, p}\left(\bar{U}_{j, i}-\bar{\Phi}_{i j}\right)\right]\right\} d A= \\
& =-\frac{d}{d x_{3}} \int_{D\left(x_{3}\right)}\left\{\left[A_{3 j m n} U_{n, m}+G_{3 j m n}\left(U_{n, m}-\Phi_{n m}\right)+F_{3 j m n r} \Phi_{n r, m}-\iota \omega D_{3 j} \mathcal{T}\right] x_{p} \bar{U}_{j, p}\right. \\
& \left.-\left[A_{3 j m n} \bar{U}_{n, m}+G_{3 j m n}\left(\bar{U}_{n, m}-\bar{\Phi}_{n m}\right)+F_{3 j m n r} \bar{\Phi}_{n r, m}+\iota \omega D_{3 j} \overline{\mathcal{T}}\right] x_{p} U_{j, p}\right\} d A \\
& -\frac{d}{d x_{3}} \int_{D\left(x_{3}\right)}\left\{\left[G_{3 j m n} U_{n, m}+B_{3 j m n}\left(U_{n, m}-\Phi_{n m}\right)+D_{3 j m n r} \Phi_{n r, m}-\iota \omega E_{3 j} \mathcal{T}\right] x_{p} \bar{U}_{j, p}\right. \\
& \left.-\left[G_{3 j m n} \bar{U}_{n, m}+B_{3 j m n}\left(\bar{U}_{n, m}-\bar{\Phi}_{n m}\right)+D_{3 j m n r} \bar{\Phi}_{n r, m}+\iota \omega E_{3 j} \overline{\mathcal{T}}\right] x_{p} U_{j, p}\right\} d A \\
& -\frac{d}{d x_{3}} \int_{D\left(x_{3}\right)}\left\{\left[F_{3 j k m n} U_{n, m}+D_{3 j k m n}\left(U_{n, m}-\Phi_{n m}\right)+C_{3 j k m n r} \Phi_{n r, m}-\iota \omega F_{3 j k} \mathcal{T}\right] x_{p} \bar{\Phi}_{j k, p}\right. \\
& \left.-\left[F_{3 j k m n} \bar{U}_{n, m}+D_{3 j k m n}\left(\bar{U}_{n, m}-\bar{\Phi}_{n m}\right)+C_{3 j k m n r} \bar{\Phi}_{n r, m}+\iota \omega F_{3 j k} \overline{\mathcal{T}}\right] x_{p} \Phi_{j k, p}\right\} d A \\
& +\frac{d}{d x_{3}} \int_{D\left(x_{3}\right)} x_{3}\left\{A_{i j m n} U_{j, i} \bar{U}_{n, m}+G_{i j m n}\left[U_{j, i}\left(\bar{U}_{n, m}-\bar{\Phi}_{n m}\right)+\bar{U}_{j, i}\left(U_{n, m}-\Phi_{n m}\right)\right]\right. \\
& +B_{i j m n}\left(U_{j, i}-\Phi_{i j}\right)\left(\bar{U}_{n, m}-\bar{\Phi}_{n m}\right)+F_{i j m n r}\left(U_{j, i} \bar{\Phi}_{n r, m}+\bar{U}_{j, i} \Phi_{n r, m}\right)- \\
& \left.+D_{i j m n r}\left[\left(\bar{U}_{j, i}-\bar{\Phi}_{i j}\right) \Phi_{n r, m}+\bar{\Phi}_{n r, m}\left(U_{j, i}-\Phi_{i j}\right)\right]+C_{i j k m n r} \Phi_{j k, i} \bar{\Phi}_{n r, m}\right\} d A+ \\
& -\frac{d}{d x_{3}} \int_{D\left(x_{3}\right)} x_{3}\left[\omega^{2}\left(\varrho U_{i} \bar{U}_{i}+I_{k r} \Phi_{j r} \bar{\Phi}_{j k}\right)+\iota \omega D_{i j}\left(\overline{\mathcal{T}} U_{j, i}-\mathcal{T} \bar{U}_{j, i}\right)\right] d A- \\
& -\iota \omega \frac{d}{d x_{3}} \int_{D\left(x_{3}\right)} x_{3}\left\{E_{i j}\left[\overline{\mathcal{T}}\left(U_{j, i}-\Phi_{i j}\right)-\mathcal{T}\left(\bar{U}_{j, i}-\bar{\Phi}_{i j}\right)\right]+F_{i j k}\left(\overline{\mathcal{T}} \Phi_{j k, i}-\mathcal{T} \bar{\Phi}_{j k, i}\right)\right\} d A+ \\
& +\int_{\partial D\left(x_{3}\right)} x_{p} n_{p}\left\{A_{i j m n} U_{j, i} \bar{U}_{n, m}+C_{i j k m n r} \Phi_{j k, i} \bar{\Phi}_{n r, m}+\right. \\
& \left.+F_{i j m n r}\left(U_{j, i} \bar{\Phi}_{n r, m}+\bar{U}_{j, i} \Phi_{n r, m}\right)\right\} d s
\end{aligned}
$$

Let us denote, as usual, with $\tau_{\alpha}$ are components of the unit vector tangent to $\partial D$, with $n_{\alpha}$ are components of the unit vector normal to $\partial D$, with $\partial / \partial \tau$ the derivative in the direction of tangent and with $\partial / \partial \alpha$ the derivative in the direction of normal. Then, on the curve $\partial D$ we have

$$
U_{i, \alpha}=n_{\alpha} \frac{\partial U_{i}}{\partial n}+\tau_{\alpha} \frac{\partial U_{i}}{\partial \tau}
$$

Taking into account the lateral boundary condition (14) we deduce $\partial U_{i} / \partial \tau=0$ 
on the curve $\partial D$ and hence we obtain

$$
\begin{aligned}
U_{i, 3} & =0 \text { on } \partial D\left(x_{3}\right) . \\
U_{i, \alpha} & =n_{\alpha} \frac{\partial U_{i}}{\partial n} \text { on } \partial D\left(x_{3}\right)
\end{aligned}
$$

The last integral in (33), with the help of relations (34), will receive the form

$$
\begin{aligned}
& \int_{\partial D\left(x_{3}\right)} x_{p} n_{p}\left(A_{i j m n} U_{j, i} \bar{U}_{n, m}+F_{i j m n r} U_{j, i} \bar{\Phi}_{n r, m}+C_{i j k m n r} \Phi_{j k, i} \bar{\Phi}_{n r, m}\right) d s= \\
& =\int_{\partial D\left(x_{3}\right)} x_{p} n_{p}\left(A_{i \alpha m \beta} \frac{\partial U_{i}}{\partial n} \frac{\partial \bar{U}_{m}}{\partial n}+2 F_{i \alpha k m \beta} \frac{\partial U_{i}}{\partial n} \frac{\partial \bar{\Phi}_{k m}}{\partial n}+C_{i j \alpha k m \beta} \frac{\partial \Phi_{i j}}{\partial n} \frac{\partial \bar{\Phi}_{k m}}{\partial n}\right) n_{\alpha} n_{\beta} d s
\end{aligned}
$$

If we substitute (35) in (33), we obtain relation (28).

We intend now to prove the relation (29). For this, we start from equation $(13)_{3}$ from where we deduce

$$
\begin{gathered}
x_{p} \overline{\mathcal{T}}_{, p}\left[\left(\frac{1}{T_{0}} K_{i j} \mathcal{T}_{, j}\right)_{, i}-\iota \omega D_{i j} U_{j, i}-\iota \omega E_{i j}\left(U_{j, i}-\Phi_{i j}\right)-\iota \omega F_{i j k} \Phi_{j k, i}+\frac{c}{T_{0}} \omega^{2} \mathcal{T}\right]+ \\
+x_{p} \mathcal{T}_{, p}\left[\left(\frac{1}{T_{0}} K_{i j} \overline{\mathcal{T}}_{, j}\right)_{, i}-\iota \omega D_{i j} \bar{U}_{j, i}-\iota \omega E_{i j}\left(\bar{U}_{j, i}-\bar{\Phi}_{i j}\right)-\iota \omega F_{i j k} \bar{\Phi}_{j k, i}+\frac{c}{T_{0}} \omega^{2} \overline{\mathcal{T}}\right]=0
\end{gathered}
$$

It's easy to rewrite equation (36) in the form

$$
\begin{aligned}
& \iota \omega D_{i j} x_{p}\left(\bar{U}_{j, i} \mathcal{T}, p-U_{j, i} \overline{\mathcal{T}}_{, p}\right)+\iota \omega E_{i j} x_{p}\left[\left(\bar{U}_{j, i}-\bar{\Phi}_{i j}\right) \mathcal{T}, p-\left(U_{j, i}-\Phi_{i j}\right) \overline{\mathcal{T}}_{, p}\right]+ \\
& +\iota \omega F_{i j k} x_{p}\left(\bar{\Phi}_{j k, i} \mathcal{T}_{, p}-\Phi_{j k, i} \overline{\mathcal{T}}_{, p}\right)=-x_{p}\left(\frac{c}{T_{0}} \omega^{2} \mathcal{T} \overline{\mathcal{T}}\right)_{, p}+\frac{2}{T_{0}} K_{i j} \mathcal{T}_{, i} \overline{\mathcal{T}}_{, j}- \\
& -\left[\frac{1}{T_{0}} x_{p} K_{i j}\left(\overline{\mathcal{T}}_{, p} \mathcal{T}_{, j}+\mathcal{T}_{, p} \overline{\mathcal{T}}_{, j}\right)\right]_{, i}+x_{p}\left(\frac{1}{T_{0}} K_{i j} \mathcal{T}_{, i} \overline{\mathcal{T}}_{, j}\right)_{, p}
\end{aligned}
$$

To facilitate the proof of relation (29), it is useful to write the equality (37) as follows

$$
\begin{aligned}
& \frac{1}{\theta_{0}} K_{i j} \mathcal{T}_{, i} \overline{\mathcal{T}}_{, j}-\frac{3 c}{\theta_{0}} \omega^{2} \mathcal{T} \overline{\mathcal{T}}+\iota \omega D_{i j} x_{p}\left(U_{j, i} \mathcal{T}_{, p}-U_{j, i} \overline{\mathcal{T}}_{, p}\right)+ \\
& +\iota \omega E_{i j} x_{p}\left[\left(\bar{U}_{j, i}-\bar{\Phi}_{i j}\right) \mathcal{T}_{, p}-\left(U_{j, i}-\Phi_{i j}\right) \overline{\mathcal{T}}_{, p}\right]+\iota \omega F_{i j k}\left(\bar{\Phi}_{j k, i} \mathcal{T}_{, p}-\Phi_{j k, i} \overline{\mathcal{T}}_{, p}\right)=(40) \\
& =-\left(\frac{c}{T_{0}} \omega^{2} \mathcal{T} \overline{\mathcal{T}}\right)_{, p}-\left[\frac{1}{T_{0}} x_{p} K_{i j}\left(\overline{\mathcal{T}}, p \mathcal{T}_{, j}+\mathcal{T}_{, p} \overline{\mathcal{T}}_{, j}\right)\right]_{, i}+\left(\frac{x_{p}}{T_{0}} K_{i j} \mathcal{T}_{, i} \overline{\mathcal{T}}_{, j}\right)_{, p}
\end{aligned}
$$

You just need to integrate equality $(38)$ on $D\left(x_{3}\right)$ and keep in mind the lateral 
boundary condition (14). So, we obtain

$$
\begin{aligned}
& \int_{D\left(x_{3}\right)} \frac{1}{T_{0}}\left(K_{i j} \mathcal{T},{ }_{i} \overline{\mathcal{T}},{ }_{j}-3 c \omega^{2} \mathcal{T} \overline{\mathcal{T}}\right) d A+\int_{D\left(x_{3}\right)} \iota \omega D_{i j} x_{p}\left(U_{j, i} \mathcal{T}, p-U_{j, i} \overline{\mathcal{T}}, p\right) d A+ \\
& +\int_{D\left(x_{3}\right)} \iota \omega\left\{E_{i j} x_{p}\left[\left(\bar{U}_{j, i}-\bar{\Phi}_{i j}\right) \mathcal{T}_{, p}-\left(U_{j, i}-\Phi_{i j}\right) \overline{\mathcal{T}}_{, p}\right]+F_{i j k}\left(\bar{\Phi}_{j k, i} \mathcal{T}_{, p}-\Phi_{j k, i} \overline{\mathcal{T}}_{, p}\right)\right\} d A= \\
& =-\frac{d}{d x_{3}} \int_{D\left(x_{3}\right)}\left[\frac{1}{T_{0}} x_{p} K_{3 j}\left(\overline{\mathcal{T}}_{, p} \mathcal{T}_{, j}+\mathcal{T}_{, p} \overline{\mathcal{T}}_{, j}\right)-\frac{x_{3}}{T_{0}} K_{i j} \mathcal{T}_{, i} \overline{\mathcal{T}}_{, j}+\frac{x_{3}}{T_{0}} c \omega^{2} \mathcal{T} \overline{\mathcal{T}}\right] d A+ \\
& +\int_{\partial D\left(x_{3}\right)} \frac{1}{T_{0}}\left[x_{p} n_{p} K_{i j} \mathcal{T}_{, i} \overline{\mathcal{T}}_{, j}-x_{p} K_{p j}\left(\overline{\mathcal{T}}_{, p} \mathcal{T}_{, j}+\mathcal{T}_{, p} \overline{\mathcal{T}}_{, j}\right) n_{p}\right] d s
\end{aligned}
$$

In equality (39) we will consider that on the curve $\partial D\left(x_{3}\right)$, we have $T,{ }_{3}=0$ and

$$
T, \alpha=n_{\alpha} \frac{\partial T}{\partial n}, \alpha=1,2 .
$$

These relations are obtained based on the lateral boundary condition (14) using a procedure analogous to that used in the proof of equality (28).

Now it is clear that the equality (39) implies the relation (29), which ends the proof of Theorem 2 .

In the following theorem we will prove two conservation laws which will be used to derive a priori estimates for a solution of our mixed problem.

Theorem 3. Let $\left(U_{i}, \Phi_{i j}, \mathcal{T}\right)$ be a solution of the boundary value problem consisting of Eqs. (13)-(15). Then, the following two conservation laws are satisfied

$$
\begin{aligned}
& \frac{d}{d x_{3}} \int_{D\left(x_{3}\right)} \omega^{2}\left(\varrho U_{j} \bar{U}_{j}+I_{k r} \Phi_{j r} \bar{\Phi}_{j k}+\frac{c}{T_{0}} \mathcal{T} \overline{\mathcal{T}}\right) d A+ \\
& +\frac{d}{d x_{3}} \int_{D\left(x_{3}\right)} \frac{c}{T_{0}}\left(K_{33} \mathcal{T}_{, 3} \overline{\mathcal{T}}_{, 3}-K_{\alpha \beta} \mathcal{T}_{, \alpha} \overline{\mathcal{T}}_{, \beta}\right) d A+ \\
& +\frac{d}{d x_{3}} \int_{D\left(x_{3}\right)}\left\{A_{i 3 m 3} U_{j, 3} \bar{U}_{m, 3}+G_{i 3 m 3}\left[U_{i, 3}\left(\bar{U}_{m, 3}-\bar{\Phi}_{m 3}\right)+\bar{U}_{i, 3}\left(U_{m, 3}-\Phi_{m 3}\right)\right]+\right. \\
& +B_{i 3 m 3}\left(U_{i, 3}-\Phi_{i 3}\right)\left(\bar{U}_{m, 3}-\bar{\Phi}_{m 3}\right)+F_{i 3 m n 3}\left(U_{i, 3} \bar{\Phi}_{m n, 3}+\bar{U}_{i, 3} \Phi_{m n, 3}\right)- \\
& \left.+D_{i 3 m n 3}\left[\left(\bar{U}_{i, 3}-\bar{\Phi}_{i 3}\right) \Phi_{m n, 3}+\bar{\Phi}_{m n, 3}\left(U_{i, 3}-\Phi_{i 3}\right)\right]+C_{i 3 k m n 3} \Phi_{i k, 3} \bar{\Phi}_{m n, 3}\right\} d A \\
& +\frac{d}{d x_{3}} \int_{D\left(x_{3}\right)}\left\{A_{i \alpha m \beta} U_{j, \alpha} \bar{U}_{m, \beta}+G_{i \alpha m \beta}\left[U_{i, \alpha}\left(\bar{U}_{m, \beta}-\bar{\Phi}_{m \beta}\right)+\bar{U}_{i, \alpha}\left(U_{m, \beta}-\Phi_{m \beta}\right)\right]\right. \\
& +B_{i \alpha m \beta}\left(U_{i, \alpha}-\Phi_{i \alpha}\right)\left(\bar{U}_{m, \beta}-\bar{\Phi}_{m \beta}\right)+F_{i \alpha m n \beta}\left(U_{i, \alpha} \bar{\Phi}_{m n, \beta}+\bar{U}_{i, \alpha} \Phi_{m n, \beta}\right)- \\
& \left.+D_{i \alpha m n \beta}\left[\left(\bar{U}_{i, \alpha}-\bar{\Phi}_{i \alpha}\right) \Phi_{m n, \beta}+\bar{\Phi}_{m n, \beta}\left(U_{i, \alpha}-\Phi_{i \alpha}\right)\right]+C_{i \alpha k m n \beta} \Phi_{i k, \alpha} \bar{\Phi}_{m n, \beta}\right\} d A \\
& +\frac{d}{d x_{3}} \int_{D\left(x_{3}\right)}\left\{\iota \omega D_{i \alpha}\left(\overline{\mathcal{T}} U_{i, \alpha}-\mathcal{T} \bar{U}_{i, \alpha}\right)+\iota \omega F_{i j \alpha}\left(\overline{\mathcal{T}} \Phi_{i j, \alpha}-\mathcal{T} \bar{\Phi}_{i j, \alpha}\right)+\right. \\
& \left.+\iota \omega E_{i \alpha}\left[\overline{\mathcal{T}}\left(U_{i, \alpha}-\Phi_{i \alpha}\right)-\mathcal{T}\left(\bar{U}_{i, \alpha}-\bar{\Phi}_{i \alpha}\right)\right]\right\} d A=0
\end{aligned}
$$




$$
\begin{gathered}
\frac{d}{d x_{3}} \int_{D\left(x_{3}\right)}\left\{\left[A_{3 j m n} \bar{U}_{n, m}+G_{3 j m n}\left(\bar{U}_{n, m}-\bar{\Phi}_{n m}\right)+F_{3 j m n r} \bar{\Phi}_{n r, m}+\iota \omega D_{3 j} \overline{\mathcal{T}}\right] U_{j}-\right. \\
\left.-\left[A_{3 j m n} U_{n, m}+G_{3 j m n}\left(U_{n, m}-\Phi_{n m}\right)+F_{3 j m n r} \Phi_{n r, m}-\iota \omega D_{3 j} \mathcal{T}\right] \bar{U}_{j}\right\} d A \\
+\frac{d}{d x_{3}} \int_{D\left(x_{3}\right)}\left[G_{3 j m n} \bar{U}_{n, m}+B_{3 j m n}\left(\bar{U}_{n, m}-\bar{\Phi}_{n m}\right)+D_{3 j m n r} \bar{\Phi}_{n r, m}+\iota \omega E_{3 j} \overline{\mathcal{T}}\right] U_{j} \\
\left.+\left[G_{3 j m n} U_{n, m}+B_{3 j m n}\left(U_{n, m}-\Phi_{n m}\right)+D_{3 j m n r} \Phi_{n r, m}-\iota \omega E_{3 j} \mathcal{T}\right] \bar{U}_{j} d A(43)\right) \\
+\frac{d}{d x_{3}} \int_{D\left(x_{3}\right)}\left[F_{3 j k m n} \bar{U}_{n, m}+D_{3 j k m n}\left(\bar{U}_{n, m}-\bar{\Phi}_{n m}\right)+C_{3 j k m n r} \bar{\Phi}_{n r, m}+\iota \omega F_{3 j k} \overline{\mathcal{T}}\right] \Phi_{j k} \\
-\left[F_{3 j k m n} U_{n, m}+D_{3 j k m n}\left(U_{n, m}-\Phi_{n m}\right)+C_{3 j k m n r} \Phi_{n r, m}-\iota \omega F_{3 j k} \mathcal{T}\right] \bar{\Phi}_{j k} d A= \\
\quad=\frac{d}{d x_{3}} \int_{D\left(x_{3}\right)}\left[\frac{1}{\theta_{0}} K_{3 j}\left(\bar{T} T T_{j}-T \bar{T}_{, j}\right)\right] d A
\end{gathered}
$$

Proof. Multiplying equation (13) 1 with $\bar{U}_{i, 3}$ and equation $(13)_{2}$ with $\bar{\Phi}_{j k, 3}$, then we add the resulting relations. We then made a similar combination regarding to the conjugates of equations $(13)_{1}$ and $(13)_{2}$. So, we obtain

$$
\begin{gathered}
\left\{\left[\left(A_{i j m n}+G_{i j m n}\right) U_{n, m}+\left(G_{m n i j}+B_{i j m n}\right)\left(U_{n, m}-\Phi_{m n}\right)+\right.\right. \\
\left.\left.+\left(F_{m n r i j}+D_{i j m n r}\right) \Phi_{n r, m}-\iota \omega\left(D_{i j}+E_{i j}\right) \mathcal{T}\right]_{, i}+\varrho \omega^{2} U_{i}\right\} \bar{U}_{j, 3}+ \\
+\left\{\left[F_{i j k m n} U_{n, m}+D_{m n i j k}\left(U_{n, m}-\Phi_{m n}\right)+C_{i j k m n r} \Phi_{n r, m}-\iota \omega F_{i j k} \mathcal{T}\right]_{, i}+\right. \\
\left.+G_{j k m n} U_{n, m}+B_{j k m n}\left(U_{n, m}-\Phi_{m n}\right)+D_{j k m n r} \Phi_{n r, m}-\iota \omega F_{j k} \mathcal{T}+I_{k r} \omega^{2} \Phi_{j r}\right\} \bar{\Phi}_{j k, 3} \\
+\left\{\left[\left(A_{i j m n}+G_{i j m n}\right) \bar{U}_{n, m}+\left(G_{m n i j}+B_{i j m n}\right)\left(\bar{U}_{n, m}-\bar{\Phi}_{m n}\right)+\right.\right. \\
\left.\left.+\left(F_{m n r i j}+D_{i j m n r}\right) \bar{\Phi}_{n r, m}+\iota \omega\left(D_{i j}+E_{i j}\right) \overline{\mathcal{T}}\right]_{, i}+\varrho \omega^{2} \bar{U}_{i}\right\} U_{j, 3}+ \\
+\left\{\left[F_{i j k m n} \bar{U}_{n, m}+D_{m n i j k}\left(\bar{U}_{n, m}-\bar{\Phi}_{m n}\right)+C_{i j k m n r} \bar{\Phi}_{n r, m}+\iota \omega F_{i j k} \overline{\mathcal{T}}\right]_{, i}+\right. \\
\left.+G_{j k m n} \bar{U}_{n, m}+B_{j k m n}\left(\bar{U}_{n, m}-\bar{\Phi}_{m n}\right)+D_{j k m n r} \bar{\Phi}_{n r, m}+\iota \omega F_{j k} \overline{\mathcal{T}}+I_{k r} \omega^{2} \bar{\Phi}_{j r}\right\} \Phi_{j k, 3}=0
\end{gathered}
$$

By direct calculations on equality (42) we obtain

$$
\begin{aligned}
& \frac{d}{d x_{3}}\left\{A_{i 3 m 3} U_{j, 3} \bar{U}_{m, 3}+G_{i 3 m 3}\left[U_{i, 3}\left(\bar{U}_{m, 3}-\bar{\Phi}_{m 3}\right)+\bar{U}_{i, 3}\left(U_{m, 3}-\Phi_{m 3}\right)\right]+\right. \\
& +B_{i 3 m 3}\left(U_{i, 3}-\Phi_{i 3}\right)\left(\bar{U}_{m, 3}-\bar{\Phi}_{m 3}\right)+F_{i 3 m n 3}\left(U_{i, 3} \bar{\Phi}_{m n, 3}+\bar{U}_{i, 3} \Phi_{m n, 3}\right)+ \\
& +D_{i 3 m n 3}\left[\left(\bar{U}_{i, 3}-\bar{\Phi}_{i 3}\right) \Phi_{m n, 3}+\bar{\Phi}_{m n, 3}\left(U_{i, 3}-\Phi_{i 3}\right)\right]+C_{i 3 k m n 3} \Phi_{i k, 3} \bar{\Phi}_{m n, 3}- \\
& -A_{i \alpha m \beta} U_{j, \alpha} \bar{U}_{m, \beta}-G_{i \alpha m \beta}\left[U_{i, \alpha}\left(\bar{U}_{m, \beta}-\bar{\Phi}_{m \beta}\right)+\bar{U}_{i, \alpha}\left(U_{m, \beta}-\Phi_{m \beta}\right)\right] \\
& -B_{i \alpha m \beta}\left(U_{i, \alpha}-\Phi_{i \alpha}\right)\left(\bar{U}_{m, \beta}-\bar{\Phi}_{m \beta}\right)-F_{i \alpha m n \beta}\left(U_{i, \alpha} \bar{\Phi}_{m n, \beta}+\bar{U}_{i, \alpha} \Phi_{m n, \beta}\right)- \\
& -D_{i \alpha m n \beta}\left[\left(\bar{U}_{i, \alpha}-\bar{\Phi}_{i \alpha}\right) \Phi_{m n, \beta}+\bar{\Phi}_{m n, \beta}\left(U_{i, \alpha}-\Phi_{i \alpha}\right)\right]-C_{i \alpha k m n \beta} \Phi_{i k, \alpha} \bar{\Phi}_{m n, \beta}+ \\
& +\iota \omega D_{i \alpha}\left(\overline{\mathcal{T}} U_{i, \alpha}-\mathcal{T} \bar{U}_{i, \alpha}\right)+\iota \omega F_{i j \alpha}\left(\overline{\mathcal{T}} \Phi_{i j, \alpha}-\mathcal{T} \bar{\Phi}_{i j, \alpha}\right)+
\end{aligned}
$$




$$
\begin{aligned}
& \left.+\iota \omega E_{i \alpha}\left[\overline{\mathcal{T}}\left(U_{i, \alpha}-\Phi_{i \alpha}\right)-\mathcal{T}\left(\bar{U}_{i, \alpha}-\bar{\Phi}_{i \alpha}\right)\right]+\varrho \omega^{2} U_{j} \bar{U}_{j}+I_{k r} \omega^{2} \Phi_{j r} \bar{\Phi}_{j k}\right\}+ \\
& +\left\{A_{i \alpha m 3} U_{m, 3} \bar{U}_{i, 3}+G_{i \alpha m 3}\left[U_{m, 3}\left(\bar{U}_{i, 3}-\bar{\Phi}_{i 3}\right)+\bar{U}_{m, 3}\left(U_{i, 3}-\Phi_{i 3}\right)\right]+\right. \\
& +B_{i \alpha m 3}\left(U_{i, 3}-\Phi_{i 3}\right)\left(\bar{U}_{m, 3}-\bar{\Phi}_{m 3}\right)+F_{i \alpha m n 3}\left(U_{i, 3} \bar{\Phi}_{m n, 3}+\bar{U}_{i, 3} \Phi_{m n, 3}\right)- \\
& +D_{i \alpha m n 3}\left[\left(\bar{U}_{i, 3}-\bar{\Phi}_{i 3}\right) \Phi_{m n, 3}+\bar{\Phi}_{m n, 3}\left(U_{i, 3}-\Phi_{i 3}\right)\right]+C_{i \alpha k m n 3} \Phi_{i k, 3} \bar{\Phi}_{m n, 3}+ \\
& +\iota \omega D_{i \alpha}\left(\overline{\mathcal{T}} U_{i, 3}-\mathcal{T} \bar{U}_{i, 3}\right)+\iota \omega F_{i j \alpha}\left(\overline{\mathcal{T}} \Phi_{i j, 3}-\mathcal{T} \bar{\Phi}_{i j, 3}\right)+ \\
& \left.+\iota \omega E_{i \alpha}\left[\overline{\mathcal{T}}\left(U_{i, 3}-\Phi_{i 3}\right)-\mathcal{T}\left(\bar{U}_{i, 3}-\bar{\Phi}_{i 3}\right)\right]\right\}_{, \alpha}+\iota \omega D_{i j}\left(\overline{\mathcal{T}},{ }_{, 3} U_{i, j}-\mathcal{T},{ }_{, 3} \bar{U}_{i, j}\right)+ \\
& +\iota \omega E_{i j}\left[\overline{\mathcal{T}}, 3\left(U_{i, j}-\Phi_{i j}\right)-\mathcal{T}, 3\left(\bar{U}_{i, j}-\bar{\Phi}_{i j}\right)\right]+\iota \omega F_{i j k}\left(\overline{\mathcal{T}}_{, 3} \Phi_{i j, k}-\mathcal{T}, 3 \bar{\Phi}_{i j, k}\right)=0 .
\end{aligned}
$$

After an integration in equality (43) over $D\left(x_{3}\right)$ and using the lateral boundary condition (14) we obtain

$$
\begin{aligned}
& \frac{d}{d x_{3}} \int_{D\left(x_{3}\right)}\left\{\varrho \omega^{2} U_{j} \bar{U}_{j}+A_{i 3 m 3} U_{j, 3} \bar{U}_{m, 3}+G_{i 3 m 3}\left[U_{i, 3}\left(\bar{U}_{m, 3}-\bar{\Phi}_{m 3}\right)+\bar{U}_{i, 3}\left(U_{m, 3}-\Phi_{m 3}\right)\right]+\right. \\
& +B_{i 3 m 3}\left(U_{i, 3}-\Phi_{i 3}\right)\left(\bar{U}_{m, 3}-\bar{\Phi}_{m 3}\right)+F_{i 3 m n 3}\left(U_{i, 3} \bar{\Phi}_{m n, 3}+\bar{U}_{i, 3} \Phi_{m n, 3}\right)+ \\
& +D_{i 3 m n 3}\left[\left(\bar{U}_{i, 3}-\bar{\Phi}_{i 3}\right) \Phi_{m n, 3}+\bar{\Phi}_{m n, 3}\left(U_{i, 3}-\Phi_{i 3}\right)\right]+C_{i 3 k m n 3} \Phi_{i k, 3} \bar{\Phi}_{m n, 3}- \\
& -A_{i \alpha m \beta} U_{j, \alpha} \bar{U}_{m, \beta}-G_{i \alpha m \beta}\left[U_{i, \alpha}\left(\bar{U}_{m, \beta}-\bar{\Phi}_{m \beta}\right)+\bar{U}_{i, \alpha}\left(U_{m, \beta}-\Phi_{m \beta}\right)\right] \\
& -B_{i \alpha m \beta}\left(U_{i, \alpha}-\Phi_{i \alpha}\right)\left(\bar{U}_{m, \beta}-\bar{\Phi}_{m \beta}\right)-F_{i \alpha m n \beta}\left(U_{i, \alpha} \bar{\Phi}_{m n, \beta}+\bar{U}_{i, \alpha} \Phi_{m n, \beta}\right)- \\
& -D_{i \alpha m n \beta}\left[\left(\bar{U}_{i, \alpha}-\bar{\Phi}_{i \alpha}\right) \Phi_{m n, \beta}+\bar{\Phi}_{m n, \beta}\left(U_{i, \alpha}-\Phi_{i \alpha}\right)\right]-C_{i \alpha k m n \beta} \Phi_{i k, \alpha} \bar{\Phi}_{m n, \beta}+ \\
& +\iota \omega D_{i \alpha}\left(\overline{\mathcal{T}} U_{i, \alpha}-\mathcal{T} \bar{U}_{i, \alpha}\right)+\iota \omega F_{i j \alpha}\left(\overline{\mathcal{T}} \Phi_{i j, \alpha}-\mathcal{T} \bar{\Phi}_{i j, \alpha}\right)+ \\
& \left.+\iota \omega E_{i \alpha}\left[\overline{\mathcal{T}}\left(U_{i, \alpha}-\Phi_{i \alpha}\right)-\mathcal{T}\left(\bar{U}_{i, \alpha}-\bar{\Phi}_{i \alpha}\right)\right]+I_{k r} \omega^{2} \Phi_{j r} \bar{\Phi}_{j k}\right\} d A+ \\
& +\int_{D\left(x_{3}\right)}\left[\iota \omega D_{i j}\left(\overline{\mathcal{T}},{ }_{3} U_{i, j}-\mathcal{T},{ }_{, 3} \bar{U}_{i, j}\right)+\iota \omega F_{i j k}\left(\overline{\mathcal{T}}_{, 3} \Phi_{i j, k}-\mathcal{T},{ }_{3} \bar{\Phi}_{i j, k}\right)\right] d A+ \\
& +\int_{D\left(x_{3}\right)} \iota \omega E_{i j}\left[\overline{\mathcal{T}}_{, 3}\left(U_{i, j}-\Phi_{i j}\right)-\mathcal{T},{ }_{, 3}\left(\bar{U}_{i, j}-\bar{\Phi}_{i j}\right)\right] d A=0
\end{aligned}
$$

With the help of equation $(13)_{3}$ we can write

$$
\begin{gathered}
\overline{\mathcal{T}}_{, 3}\left[\frac{1}{\theta_{0}} K_{i j} \mathcal{T}_{, i j}-\iota \omega D_{i j} U_{i, j}-\iota \omega E_{i j}\left(U_{i, j}-\Phi_{i, j}\right)-\iota \omega F_{i j k} \Phi_{j k, i}+\frac{c}{T_{0}} \omega^{2} T\right]+ \\
+\mathcal{T}_{, 3}\left[\frac{1}{\theta_{0}} K_{i j} \overline{\mathcal{T}}_{, i j}+\iota \omega D_{i j} \bar{U}_{i, j}+\iota \omega E_{i j}\left(\bar{U}_{i, j}-\bar{\Phi}_{i, j}\right)+\iota \omega F_{i j k} \bar{\Phi}_{j k, i}+\frac{c}{T_{0}} \omega^{2} \overline{\mathcal{T}}\right]=0
\end{gathered}
$$

and this relation can be rewritten in the form

$$
\begin{aligned}
& \frac{d}{d x_{3}}\left(\frac{c}{T_{0}} \omega^{2} \mathcal{T} \overline{\mathcal{T}}+\frac{1}{T_{0}} K_{33} \mathcal{T}_{, 3} \overline{\mathcal{T}}_{, 3}-\frac{1}{T_{0}} K_{\alpha \beta} \mathcal{T}_{, \alpha} \overline{\mathcal{T}}_{, \beta}\right)+\left(\frac{2}{T_{0}} K_{\alpha 3} \mathcal{T}_{, 3} \overline{\mathcal{T}}_{, 3}\right)_{, \alpha}+ \\
& \quad+\iota \omega D_{i j}\left(\mathcal{T},{ }_{3} \bar{U}_{i, j}-\overline{\mathcal{T}}_{, 3} U_{i, j}\right)+\iota \omega F_{i j k}\left(\mathcal{T}_{, 3} \bar{\Phi}_{j k, i}-\overline{\mathcal{T}},{ }_{3} \Phi_{j k, i}\right)+ \\
& \quad+\iota \omega E_{i j}\left[\mathcal{T}_{, 3}\left(\bar{U}_{i, j}-\bar{\Phi}_{i j}\right)-\overline{\mathcal{T}}_{, 3}\left(U_{i, j}-\Phi_{i j}\right)\right]=0
\end{aligned}
$$


Now we integrate (46) over $D\left(x_{3}\right)$ and use the lateral boundary condition (14), and thus we get

$$
\begin{aligned}
& \frac{d}{d x_{3}} \int_{D\left(x_{3}\right)}\left(\frac{c}{T_{0}} \omega^{2} \mathcal{T} \overline{\mathcal{T}}+\frac{1}{T_{0}} K_{33} \mathcal{T}_{, 3} \overline{\mathcal{T}}_{, 3}-\frac{1}{T_{0}} K_{\alpha \beta} \mathcal{T}_{, \alpha} \overline{\mathcal{T}}_{, \beta}\right) d A+ \\
& +\int_{D\left(x_{3}\right)}\left[\iota \omega D_{i j}\left(\mathcal{T}{ }_{3} \bar{U}_{i, j}-\overline{\mathcal{T}}_{, 3} U_{i, j}\right)+\iota \omega F_{i j k}\left(\mathcal{T},{ }_{3} \bar{\Phi}_{j k, i}-\overline{\mathcal{T}}_{, 3} \Phi_{j k, i}\right)\right] d A+ \\
& \quad+\int_{D\left(x_{3}\right)} \iota \omega E_{i j}\left[\mathcal{T}_{, 3}\left(\bar{U}_{i, j}-\bar{\Phi}_{i j}\right)-\overline{\mathcal{T}}_{, 3}\left(U_{i, j}-\Phi_{i j}\right)\right] d A=0
\end{aligned}
$$

Conservation Law (40) is obtained now by combining relations (44) and (47). Finally, by combining the relations (17) and (19) we obtain the conservation law (41) and this concludes the proof of Theorem 3.

In what follows we combine the relations (16) - (19) with relations (28) (29) and relation (40) - (41) in order to obtain certain measures associated to solution $\left(U_{i}, \Phi_{i j}, \mathcal{T}\right)$ of the problem (13)-(15). These measures are used to obtain suitable estimates which describe the spatial evolution of the amplitude $\left(U_{i}, \Phi_{i j}, \mathcal{T}\right)$

The first of these estimates will be proved in the following theorem.

Theorem 4. Let $\left(U_{i}, \Phi_{i}, T\right)$ be a solution of the boundary value problem consisting of Eqs. (13)-(15). Then the following equality holds

$$
\begin{array}{r}
\int_{D\left(x_{3}\right)}\left\{A_{i j m n} U_{j, i} \bar{U}_{n, m}+G_{i j m n}\left[U_{i, j}\left(\bar{U}_{n, m}-\bar{\Phi}_{m n}\right)+\bar{U}_{i, j}\left(U_{n, m}-\Phi_{m n}\right)\right]+\right. \\
+B_{i j m n}\left(U_{i, j}-\Phi_{i j}\right)\left(\bar{U}_{n, m}-\bar{\Phi}_{m n}\right)+F_{i j m n r}\left(U_{i, j} \bar{\Phi}_{m n, r}+\bar{U}_{i, j} \Phi_{m n, 3}\right)- \\
\left.+D_{i j m n r}\left[\left(\bar{U}_{i, j}-\bar{\Phi}_{i j}\right) \Phi_{m n, r}+\bar{\Phi}_{m n, r}\left(U_{i, j}-\Phi_{i j}\right)\right]+C_{i j k m n r} \Phi_{j k, i} \bar{\Phi}_{n r, m}\right\} d A- \\
\quad-\int_{D\left(x_{3}\right)}\left[\omega^{2}\left(\varrho U_{i} \bar{U}_{i}+I_{k r} \Phi_{j r} \bar{\Phi}_{j k}+\frac{c}{T_{0}} \mathcal{T} \overline{\mathcal{T}}\right)-\frac{c}{T_{0}} K_{i j} \mathcal{T},{ }_{i} \overline{\mathcal{T}}, j\right] d A+ \\
+\int_{D\left(x_{3}\right)}\left[\iota \omega D_{i j}\left(\mathcal{T} \bar{U}_{i, j}-\overline{\mathcal{T}}_{i, j}\right)+\iota \omega F_{i j k}\left(\mathcal{T} \bar{\Phi}_{j k, i}-\overline{\mathcal{T}} \Phi_{j k, i}\right)\right] d A+ \\
+\int_{D\left(x_{3}\right)} \iota \omega E_{i j}\left[\mathcal{T}\left(\bar{U}_{i, j}-\bar{\Phi}_{i j}\right)-\overline{\mathcal{T}}\left(U_{i, j}-\Phi_{i j}\right)\right] d A= \\
\frac{d}{d x_{3}} \int_{D\left(x_{3}\right)}\left\{\left[A_{3 j m n} U_{n, m}+G_{3 j m n}\left(U_{n, m}-\Phi_{n m}\right)+F_{3 j m n r} \Phi_{n r, m}-\iota \omega D_{3 j} \mathcal{T}\right] \bar{U}_{j, p}+\right. \\
\left.+\left[A_{3 j m n} \bar{U}_{n, m}+G_{3 j m n}\left(\bar{U}_{n, m}-\bar{\Phi}_{n m}\right)+F_{3 j m n r} \bar{\Phi}_{n r, m}+\iota \omega D_{3 j} \overline{\mathcal{T}}\right] U_{j, p}\right\} d A+ \\
+\frac{d}{d x_{3}} \int_{D\left(x_{3}\right)}\left\{\left[G_{3 j m n} U_{n, m}+B_{3 j m n}\left(U_{n, m}-\Phi_{n m}\right)+D_{3 j m n r} \Phi_{n r, m}-\iota \omega E_{3 j} \mathcal{T}\right] \bar{U}_{j, p}+\right. \\
\left.+\left[G_{3 j m n} \bar{U}_{n, m}+B_{3 j m n}\left(\bar{U}_{n, m}-\bar{\Phi}_{n m}\right)+D_{3 j m n r} \bar{\Phi}_{n r, m}+\iota \omega E_{3 j} \overline{\mathcal{T}}\right] U_{j, p}\right\} d A+
\end{array}
$$




$$
\begin{gathered}
+\frac{d}{d x_{3}} \int_{D\left(x_{3}\right)}\left\{\left[F_{3 j k m n} U_{n, m}+D_{3 j k m n}\left(U_{n, m}-\Phi_{n m}\right)+C_{3 j k m n r} \Phi_{n r, m}-\iota \omega F_{3 j k} \mathcal{T}\right] \bar{\Phi}_{j k, p}+\right. \\
\left.+\left[F_{3 j k m n} \bar{U}_{n, m}+D_{3 j k m n}\left(\bar{U}_{n, m}-\bar{\Phi}_{n m}\right)+C_{3 j k m n r} \bar{\Phi}_{n r, m}+\iota \omega F_{3 j k} \overline{\mathcal{T}}\right] \Phi_{j k, p}\right\} d A+ \\
+\frac{d}{d x_{3}} \int_{D\left(x_{3}\right)} \frac{1}{\theta_{0}} K_{33}\left(T \bar{T}_{, 3}+\bar{T} T,{ }_{3}\right) d A
\end{gathered}
$$

Proof. This identity is immediately obtained by combining relations (16) and (18).

In the next theorem we will prove another a priori estimates.

Theorem 5. If $\left(U_{i}, \Phi_{i j}, \mathcal{T}\right)$ is a solution of the boundary value problem consisting of Eqs. (13)-(15), then we have

$$
\begin{aligned}
& \int_{D\left(x_{3}\right)}\left\{A_{i j m n} U_{j, i} \bar{U}_{n, m}+G_{i j m n}\left[U_{i, j}\left(\bar{U}_{n, m}-\bar{\Phi}_{m n}\right)+\bar{U}_{i, j}\left(U_{n, m}-\Phi_{m n}\right)\right]+\right. \\
& +B_{i j m n}\left(U_{i, j}-\Phi_{i j}\right)\left(\bar{U}_{n, m}-\bar{\Phi}_{m n}\right)+F_{i j m n r}\left(U_{i, j} \bar{\Phi}_{m n, r}+\bar{U}_{i, j} \Phi_{m n, 3}\right)- \\
& \left.+D_{i j m n r}\left[\left(\bar{U}_{i, j}-\bar{\Phi}_{i j}\right) \Phi_{m n, r}+\bar{\Phi}_{m n, r}\left(U_{i, j}-\Phi_{i j}\right)\right]+C_{i j k m n r} \Phi_{j k, i} \bar{\Phi}_{n r, m}\right\} d A- \\
& -\int_{D\left(x_{3}\right)}\left[\omega^{2}\left(\varrho U_{i} \bar{U}_{i}+I_{k r} \Phi_{j r} \bar{\Phi}_{j k}+\frac{c}{T_{0}} \mathcal{T} \overline{\mathcal{T}}\right)-\frac{c}{T_{0}} K_{i j} \mathcal{T}_{, i} \overline{\mathcal{T}}_{, j}\right] d A- \\
& -\int_{\partial D\left(x_{3}\right)} x_{p} n_{p}\left(A_{i \alpha m \beta} \frac{\partial U_{i}}{\partial n} \frac{\partial \bar{U}_{m}}{\partial n}+2 F_{i \alpha k m \beta} \frac{\partial U_{i}}{\partial n} \frac{\partial \bar{\Phi}_{k m}}{\partial n}+C_{i j \alpha k m \beta} \frac{\partial \Phi_{i j}}{\partial n} \frac{\partial \bar{\Phi}_{k m}}{\partial n}\right) n_{\alpha} n_{\beta} d s \\
& -\int_{\partial D\left(x_{3}\right)} \frac{1}{T_{0}} x_{p} n_{p} K_{\alpha \beta} n_{\alpha} n_{\beta} \frac{\partial \mathcal{T}}{\partial n} \frac{\partial \overline{\mathcal{T}}}{\partial n} d s= \\
& =\frac{d}{d x_{3}} \int_{D\left(x_{3}\right)}\left\{\left[A_{3 j m n} U_{n, m}+G_{3 j m n}\left(U_{n, m}-\Phi_{n m}\right)+F_{3 j m n r} \Phi_{n r, m}-\iota \omega D_{3 j} \mathcal{T}\right]\left(\bar{U}_{j}+x_{p} \bar{U}_{j, p}\right)\right. \\
& \left.+\left[A_{3 j m n} \bar{U}_{n, m}+G_{3 j m n}\left(\bar{U}_{n, m}-\bar{\Phi}_{n m}\right)+F_{3 j m n r} \bar{\Phi}_{n r, m}+\iota \omega D_{3 j} \overline{\mathcal{T}}\right]\left(U_{j}+x_{p} U_{j, p}\right)\right\} d A \\
& +\left[G_{3 j m n} U_{n, m}+B_{3 j m n}\left(U_{n, m}-\Phi_{n m}\right)+D_{3 j m n r} \Phi_{n r, m}-\iota \omega E_{3 j} \mathcal{T}\right]\left(\bar{U}_{j}+x_{p} \bar{U}_{j, p}\right)+ \\
& \left.+\left[G_{3 j m n} \bar{U}_{n, m}+B_{3 j m n}\left(\bar{U}_{n, m}-\bar{\Phi}_{n m}\right)+D_{3 j m n r} \bar{\Phi}_{n r, m}+\iota \omega E_{3 j} \overline{\mathcal{T}}\right]\left(U_{j}+x_{p} U_{j, p}\right)\right\} d A \\
& +\left[F_{3 j k m n} U_{n, m}+D_{3 j k m n}\left(U_{n, m}-\Phi_{n m}\right)+C_{3 j k m n r} \Phi_{n r, m}-\iota \omega F_{3 j k} \mathcal{T}\right]\left(\bar{\Phi}_{j k}+x_{p} \bar{\Phi}_{j k, p}\right) \\
& \left.+\left[F_{3 j k m n} \bar{U}_{n, m}+D_{3 j k m n}\left(\bar{U}_{n, m}-\bar{\Phi}_{n m}\right)+C_{3 j k m n r} \bar{\Phi}_{n r, m}+\iota \omega F_{3 j k} \overline{\mathcal{T}}\right]\left(\Phi_{j k}+x_{p} \Phi_{j k, p}\right)\right\} d A \\
& +\frac{d}{d x_{3}} \int_{D\left(x_{3}\right)} \frac{1}{T_{0}} K_{33}\left(\mathcal{T} \overline{\mathcal{T}}_{, 3}+\overline{\mathcal{T}} \mathcal{T}_{, 3}\right) d A+ \\
& +\frac{d}{d x_{3}} \int_{D\left(x_{3}\right)} \frac{x_{\alpha}}{T_{0}}\left[K_{3 \alpha}\left(\overline{\mathcal{T}}, \alpha \mathcal{T}_{, \beta}+\mathcal{T}, \alpha \overline{\mathcal{T}}_{, \beta}\right)+K_{33}\left(\overline{\mathcal{T}}, \alpha \mathcal{T}_{, 3}+\mathcal{T}, \alpha \overline{\mathcal{T}}_{, 3}\right)\right] d A+ \\
& +\frac{d}{d x_{3}} \int_{D\left(x_{3}\right)} x_{3}\left\{A_{i 3 m 3} U_{j, 3} \bar{U}_{m, 3}+G_{i 3 m 3}\left[U_{i, 3}\left(\bar{U}_{m, 3}-\bar{\Phi}_{m 3}\right)+\bar{U}_{i, 3}\left(U_{m, 3}-\Phi_{m 3}\right)\right]+\right. \\
& +B_{i 3 m 3}\left(U_{i, 3}-\Phi_{i 3}\right)\left(\bar{U}_{m, 3}-\bar{\Phi}_{m 3}\right)+F_{i 3 m n 3}\left(U_{i, 3} \bar{\Phi}_{m n, 3}+\bar{U}_{i, 3} \Phi_{m n, 3}\right)+
\end{aligned}
$$


$+D_{i 3 m n 3}\left[\left(\bar{U}_{i, 3}-\bar{\Phi}_{i 3}\right) \Phi_{m n, 3}+\bar{\Phi}_{m n, 3}\left(U_{i, 3}-\Phi_{i 3}\right)\right]+C_{i 3 k m n 3} \Phi_{i k, 3} \bar{\Phi}_{m n, 3}+$
$+A_{i \alpha m \beta} U_{j, \alpha} \bar{U}_{m, \beta}+G_{i \alpha m \beta}\left[U_{i, \alpha}\left(\bar{U}_{m, \beta}-\bar{\Phi}_{m \beta}\right)+\bar{U}_{i, \alpha}\left(U_{m, \beta}-\Phi_{m \beta}\right)\right]$
$+B_{i \alpha m \beta}\left(U_{i, \alpha}-\Phi_{i \alpha}\right)\left(\bar{U}_{m, \beta}-\bar{\Phi}_{m \beta}\right)+F_{i \alpha m n \beta}\left(U_{i, \alpha} \bar{\Phi}_{m n, \beta}+\bar{U}_{i, \alpha} \Phi_{m n, \beta}\right)+$
$+D_{i \alpha m n \beta}\left[\left(\bar{U}_{i, \alpha}-\bar{\Phi}_{i \alpha}\right) \Phi_{m n, \beta}+\bar{\Phi}_{m n, \beta}\left(U_{i, \alpha}-\Phi_{i \alpha}\right)\right]+C_{i \alpha k m n \beta} \Phi_{i k, \alpha} \bar{\Phi}_{m n, \beta}-$
$-\iota \omega D_{i \alpha}\left(\overline{\mathcal{T}} U_{i, \alpha}-\mathcal{T} \bar{U}_{i, \alpha}\right)-\iota \omega F_{i j \alpha}\left(\overline{\mathcal{T}} \Phi_{i j, \alpha}-\mathcal{T} \bar{\Phi}_{i j, \alpha}\right)-$
$\left.-\iota \omega E_{i \alpha}\left[\overline{\mathcal{T}}\left(U_{i, \alpha}-\Phi_{i \alpha}\right)-\mathcal{T}\left(\bar{U}_{i, \alpha}-\bar{\Phi}_{i \alpha}\right)\right]\right\} d A+$
$+\frac{d}{d x_{3}} \int_{D\left(x_{3}\right)}\left[\frac{x_{3}}{T_{0}}\left(K_{33} \mathcal{T}_{, 3} \overline{\mathcal{T}}_{, 3}-K_{\alpha \beta} \mathcal{T}_{, \alpha} \overline{\mathcal{T}}_{, \beta}\right)+x_{3} \omega^{2}\left(U_{j} \bar{U}_{j}+I_{k r} \Phi_{j r} \bar{\Phi}_{j k}+\frac{c}{T_{0}} \mathcal{T} \overline{\mathcal{T}}\right)\right] d A$

Proof. The equality (49) can be obtained if we combine the results from equalities (28) and (29) of Theorem 2 with relation (48) of Theorem 4.

Spatial evolution of the solution of mixed problem will be studied using equality (49). To this aim we specify assumptions, which are really common in continuum mechanics. First, we assume that tensors of dipolar thermoelasticity satisfy the strong ellipticity condition

$$
\begin{aligned}
& A_{i j m n} x_{i} x_{m} y_{j} y_{n}>0, B_{i j m n} x_{i} x_{m} y_{j} y_{n}>0, \\
& \forall \text { non-zero vectors }\left(x_{1}, x_{2}, x_{3}\right),\left(y_{1}, y_{2}, y_{3}\right) \\
& C_{i j k m n r} x_{i} x_{m} y_{j} y_{n} z_{k} z_{r}>0, \\
& \forall \text { non-zero vectors }\left(x_{1}, x_{2}, x_{3}\right),\left(y_{1}, y_{2}, y_{3}\right),\left(z_{1}, z_{2}, z_{3}\right)
\end{aligned}
$$

Also, we suppose that the conductivity tensor $K_{i j}$ and the specific heat $c$ are positive, that is, satisfy the conditions

$$
c>0, K_{i j} x_{i} x_{j}>0, \text { for all non-zero vectors }\left(x_{1}, x_{2}, x_{3}\right)
$$

As a particular case, from (50) we can deduce

$$
\begin{aligned}
& A_{i 3 m 3} x_{i} x_{m}>0, B_{i 3 m 3} x_{i} x_{m}>0, \forall \text { non-zero vectors }\left(x_{1}, x_{2}, x_{3}\right) \\
& C_{i 3 k m n 3} x_{i} x_{m} y_{k} y_{n}>0, \forall \text { non-zero vectors }\left(x_{1}, x_{2}, x_{3}\right),\left(y_{1}, y_{2}, y_{3}\right)
\end{aligned}
$$

The curve $\partial D$ is regular enough that we can deduce that there is $h_{0}>0$ such that $x_{p} n_{p} \geq h_{0}>0$. So, by using the notations

$$
\begin{aligned}
& C=\left(A_{i \alpha m \beta} A_{i \alpha m \beta}+2 B_{i \alpha m \beta} B_{i \alpha m \beta}+C_{i \alpha m \beta} C_{i \alpha m \beta}\right)^{1 / 2} \\
& M=\sup _{\left(x_{1}, x_{2}\right) \in \partial D} \sqrt{\left(x_{1}^{2}+x_{2}^{2}\right)}
\end{aligned}
$$


we can deduce the inequalities

$$
\begin{aligned}
& 0 \leq \int_{\partial D\left(x_{3}\right)} x_{p} n_{p}\left(A_{i \alpha m \beta} \frac{\partial U_{i}}{\partial n} \frac{\partial \bar{U}_{m}}{\partial n}+2 F_{i \alpha k m \beta} \frac{\partial U_{i}}{\partial n} \frac{\partial \bar{\Phi}_{k m}}{\partial n}+C_{i j \alpha k m \beta} \frac{\partial \Phi_{i j}}{\partial n} \frac{\partial \bar{\Phi}_{k m}}{\partial n}\right) n_{\alpha} n_{\beta} d s \\
& \leq M C \int_{\partial D\left(x_{3}\right)}\left(\frac{\partial U_{i}}{\partial n} \frac{\partial \bar{U}_{i}}{\partial n}+\frac{\partial \Phi_{i j}}{\partial n} \frac{\partial \bar{\Phi}_{i j}}{\partial n}\right) d s
\end{aligned}
$$

Regarding the conductivity tensor $K_{i j}$, we introduce the notation

$$
K=\left(K_{\alpha \beta} K_{\alpha \beta}\right)^{1 / 2}
$$

such that we have

$$
0 \leq \int_{\partial D\left(x_{3}\right)} \frac{1}{T_{0}} x_{p} n_{p} K_{\alpha \beta} n_{\alpha} n_{\beta} \frac{\partial \mathcal{T}}{\partial n} \frac{\partial \overline{\mathcal{T}}}{\partial n} d s \leq \frac{M K}{T_{0}} \int_{\partial D\left(x_{3}\right)} \frac{\partial \mathcal{T}}{\partial n} \frac{\partial \overline{\mathcal{T}}}{\partial n} d s
$$

where $M$ is defined in (54).

In order to obtain an explicit critical value for the frequency of vibration, we will need the quantities $m_{0}, m_{1}, \omega_{0}^{*}$ and $\omega_{1}^{*}$ defined by

$$
\begin{aligned}
& m_{0}=\max _{x_{3} \in[0, L]} \frac{\int_{\partial D\left(x_{3}\right)}\left(\frac{\partial U_{i}}{\partial n} \frac{\partial \bar{U}_{m}}{\partial n}+\frac{\partial \Phi_{i j}}{\partial n} \frac{\partial \bar{\Phi}_{i j}}{\partial n}\right) d s}{\int_{D\left(x_{3}\right)}\left(U_{i} \bar{U}_{i}+\Phi_{i j} \bar{\Phi}_{i j}\right) d s}, \omega_{0}^{*}=\frac{1}{\varrho} M C m_{0} \\
& m_{1}=\max _{x_{3} \in[0, L]} \frac{\int_{\partial D\left(x_{3}\right)} \frac{\partial \mathcal{T}}{\partial n} \frac{\partial \overline{\mathcal{T}}}{\partial n} d s}{\int_{D\left(x_{3}\right)} \mathcal{T} \overline{\mathcal{T}} d s}, \omega_{1}^{*}=\frac{1}{c} M K m_{1}
\end{aligned}
$$

We always can suppose that

$$
\begin{aligned}
& \omega>\omega^{*}=\max \left\{\omega_{0}^{*}, \omega_{1}^{*}\right\} \\
& m_{0} \leq m_{0}^{*}, m_{1} \leq m_{1}^{*}
\end{aligned}
$$

where

$$
m_{0}^{*}=\max \frac{\int_{\partial D\left(x_{3}\right)}\left(\frac{\partial U_{i}}{\partial n} \frac{\partial \bar{U}_{i}}{\partial n}+\frac{\partial \Phi_{i j}}{\partial n} \frac{\partial \bar{\Phi}_{i j}}{\partial n}\right) d s}{\int_{D\left(x_{3}\right)}\left(U_{i} \bar{U}_{i}+\Phi_{i j} \bar{\Phi}_{i j}\right) d s}
$$

the maximum being calculated for $U_{i} \in H_{0}^{1}(D), \Phi_{i j} \in H_{0}^{1}(D), i, j=1,2,3$.

$$
m_{1}^{*}=\max _{\mathcal{T} \in H_{0}^{1}(D)} \frac{\int_{\partial D\left(x_{3}\right)} \frac{\partial \mathcal{T}}{\partial n} \frac{\partial \overline{\mathcal{T}}}{\partial n} d s}{\int_{D\left(x_{3}\right)} \mathcal{T} \overline{\mathcal{T}} d s}
$$


Here $H_{0}^{1}(D)$ is the usual Sobolev space. So we got an explicit critical value for the frequency of vibration, namely

$$
\omega^{*}=\max \left\{\frac{1}{\varrho} M C m_{0}^{*}, \frac{1}{c} M K m_{1}^{*}\right\}
$$

With the help of relations (49), (55) and (57) we obtain the following result regarding the spatial evolution of the amplitude $\left(U_{i}, \Phi_{i j}, \mathcal{T}\right)$

$$
\begin{aligned}
& \frac{d}{d x_{3}} \int_{D\left(x_{3}\right)}\left\{\left[A_{3 j m n} U_{n, m}+G_{3 j m n}\left(U_{n, m}-\Phi_{n m}\right)+F_{3 j m n r} \Phi_{n r, m}-\iota \omega D_{3 j} \mathcal{T}\right]\left(\bar{U}_{j}+x_{p} \bar{U}_{j, p}\right)\right. \\
& \left.+\left[A_{3 j m n} \bar{U}_{n, m}+G_{3 j m n}\left(\bar{U}_{n, m}-\bar{\Phi}_{n m}\right)+F_{3 j m n r} \bar{\Phi}_{n r, m}+\iota \omega D_{3 j} \overline{\mathcal{T}}\right]\left(U_{j}+x_{p} U_{j, p}\right)\right\} d A \\
& +\left[G_{3 j m n} U_{n, m}+B_{3 j m n}\left(U_{n, m}-\Phi_{n m}\right)+D_{3 j m n r} \Phi_{n r, m}-\iota \omega E_{3 j} \mathcal{T}\right]\left(\bar{U}_{j}+x_{p} \bar{U}_{j, p}\right)+ \\
& \left.+\left[G_{3 j m n} \bar{U}_{n, m}+B_{3 j m n}\left(\bar{U}_{n, m}-\bar{\Phi}_{n m}\right)+D_{3 j m n r} \bar{\Phi}_{n r, m}+\iota \omega E_{3 j} \overline{\mathcal{T}}\right]\left(U_{j}+x_{p} U_{j, p}\right)\right\} d A \\
& +\left[F_{3 j k m n} U_{n, m}+D_{3 j k m n}\left(U_{n, m}-\Phi_{n m}\right)+C_{3 j k m n r} \Phi_{n r, m}-\iota \omega F_{3 j k} \mathcal{T}\right]\left(\bar{\Phi}_{j k}+x_{p} \bar{\Phi}_{j k, p}\right) \\
& \left.+\left[F_{3 j k m n} \bar{U}_{n, m}+D_{3 j k m n}\left(\bar{U}_{n, m}-\bar{\Phi}_{n m}\right)+C_{3 j k m n r} \bar{\Phi}_{n r, m}+\iota \omega F_{3 j k} \overline{\mathcal{T}}\right]\left(\Phi_{j k}+x_{p} \Phi_{j k, p}\right)\right\} d A \\
& +\frac{d}{d x_{3}} \int_{D\left(x_{3}\right)}\left[\frac{1}{T_{0}} K_{33}\left(\mathcal{T} \overline{\mathcal{T}}_{, 3}+\overline{\mathfrak{T} \mathcal{T}}, 3\right)+x_{3} \omega^{2}\left(U_{j} \bar{U}_{j}+I_{k r} \Phi_{j r} \bar{\Phi}_{j k}+\frac{c}{T_{0}} \mathcal{T} \overline{\mathcal{T}}\right)\right] d A+ \\
& +\frac{d}{d x_{3}} \int_{D\left(x_{3}\right)} \frac{x_{\alpha}}{T_{0}}\left[K_{3 \alpha}\left(\overline{\mathcal{T}}_{, \alpha} \mathcal{T}_{, \beta}+\mathcal{T}_{, \alpha} \overline{\mathcal{T}}_{, \beta}\right)+K_{33}\left(\overline{\mathcal{T}}_{, \alpha} \mathcal{T}_{, 3}+\mathcal{T}_{, \alpha} \overline{\mathcal{T}}_{, 3}\right)\right] d A+ \\
& +\frac{d}{d x_{3}} \int_{D\left(x_{3}\right)} x_{3}\left\{A_{i 3 m 3} U_{j, 3} \bar{U}_{m, 3}+G_{i 3 m 3}\left[U_{i, 3}\left(\bar{U}_{m, 3}-\bar{\Phi}_{m 3}\right)+\bar{U}_{i, 3}\left(U_{m, 3}-\Phi_{m 3}\right)\right]+\right. \\
& +B_{i 3 m 3}\left(U_{i, 3}-\Phi_{i 3}\right)\left(\bar{U}_{m, 3}-\bar{\Phi}_{m 3}\right)+F_{i 3 m n 3}\left(U_{i, 3} \bar{\Phi}_{m n, 3}+\bar{U}_{i, 3} \Phi_{m n, 3}\right)+ \\
& +D_{i 3 m n 3}\left[\left(\bar{U}_{i, 3}-\bar{\Phi}_{i 3}\right) \Phi_{m n, 3}+\bar{\Phi}_{m n, 3}\left(U_{i, 3}-\Phi_{i 3}\right)\right]+C_{i 3 k m n 3} \Phi_{i k, 3} \bar{\Phi}_{m n, 3}- \\
& -A_{i \alpha m \beta} U_{j, \alpha} \bar{U}_{m, \beta}-G_{i \alpha m \beta}\left[U_{i, \alpha}\left(\bar{U}_{m, \beta}-\bar{\Phi}_{m \beta}\right)+\bar{U}_{i, \alpha}\left(U_{m, \beta}-\Phi_{m \beta}\right)\right]- \\
& -B_{i \alpha m \beta}\left(U_{i, \alpha}-\Phi_{i \alpha}\right)\left(\bar{U}_{m, \beta}-\bar{\Phi}_{m \beta}\right)-F_{i \alpha m n \beta}\left(U_{i, \alpha} \bar{\Phi}_{m n, \beta}+\bar{U}_{i, \alpha} \Phi_{m n, \beta}\right)- \\
& -D_{i \alpha m n \beta}\left[\left(\bar{U}_{i, \alpha}-\bar{\Phi}_{i \alpha}\right) \Phi_{m n, \beta}+\bar{\Phi}_{m n, \beta}\left(U_{i, \alpha}-\Phi_{i \alpha}\right)\right]-C_{i \alpha k m n \beta} \Phi_{i k, \alpha} \bar{\Phi}_{m n, \beta}- \\
& -\iota \omega D_{i \alpha}\left(\overline{\mathcal{T}} U_{i, \alpha}-\mathcal{T} \bar{U}_{i, \alpha}\right)-\imath \omega F_{i j \alpha}\left(\overline{\mathcal{T}} \Phi_{i j, \alpha}-\mathcal{T} \bar{\Phi}_{i j, \alpha}\right)- \\
& \left.-\iota \omega E_{i \alpha}\left[\overline{\mathcal{T}}\left(U_{i, \alpha}-\Phi_{i \alpha}\right)-\mathcal{T}\left(\bar{U}_{i, \alpha}-\bar{\Phi}_{i \alpha}\right)\right]\right\} d A+ \\
& +\frac{d}{d x_{3}} \int_{D\left(x_{3}\right)}\left[\frac{x_{3}}{T_{0}}\left(K_{33} \mathcal{T}_{, 3} \overline{\mathcal{T}}_{, 3}-K_{\alpha \beta} \mathcal{T}_{, \alpha} \overline{\mathcal{T}}_{, \beta}\right)+x_{3} \omega^{2}\left(U_{j} \bar{U}_{j}+I_{k r} \Phi_{j r} \bar{\Phi}_{j k}+\frac{c}{T_{0}} \mathcal{T} \overline{\mathcal{T}}\right)\right] d A
\end{aligned}
$$




$$
\begin{aligned}
& \geq \int_{D\left(x_{3}\right)}\left\{A_{i j m n} U_{j, i} \bar{U}_{n, m}+G_{i j m n}\left[U_{i, j}\left(\bar{U}_{n, m}-\bar{\Phi}_{m n}\right)+\bar{U}_{i, j}\left(U_{n, m}-\Phi_{m n}\right)\right]+\right. \\
& +B_{i j m n}\left(U_{i, j}-\Phi_{i j}\right)\left(\bar{U}_{n, m}-\bar{\Phi}_{m n}\right)+F_{i j m n r}\left(U_{i, j} \bar{\Phi}_{m n, r}+\bar{U}_{i, j} \Phi_{m n, 3}\right)- \\
& \left.+D_{i j m n r}\left[\left(\bar{U}_{i, j}-\bar{\Phi}_{i j}\right) \Phi_{m n, r}+\bar{\Phi}_{m n, r}\left(U_{i, j}-\Phi_{i j}\right)\right]+C_{i j k m n r} \Phi_{j k, i} \bar{\Phi}_{n r, m}\right\} d A+ \\
& +\int_{D\left(x_{3}\right)} \frac{1}{T_{0}} K_{i j} \mathcal{T}_{, i} \overline{\mathcal{T}}_{, j} d A
\end{aligned}
$$

Conclusion. It is easy to ascertain that the differential inequality (64) is different from those used for deduction of estimates of Saint-Venant type. Also, it is appropriate to note that to deduct these estimates we used only the strong ellipticity assumptions for the thermoelastic coefficients.

For this reason, we belive the these results can be apply for a large scale of materials.

\section{References}

[1] D.S. Chandrasekharaiah, Hyperbolic Thermoelasticity: A Review of Recent Literature, Appl. Mech. Rev., 51: 705-729, 1998.

[2] D.S. Chandrasekharaiah, A Note on the Uniqueness of Solution in the Linear Theory of Thermoelasticity Without Energy Dissipation, J. Elasticity, 43: 279-283, 1996.

[3] S. Chirita, Spatial decay estimates for solutions describing harmonic vibrations in a thermoelastic cylinder, J. Thermal Stresses, 18: 421-436, 1995.

[4] M. Ciarletta, A theory of micropolar thermoelasticity without energy dissipation, J. Thermal Stresses, 22: 581-594, 1999.

[5] C. Flaut, D. Savin, Some examples of division symbol algebras of degree 3 and 5, Carpathian Journal of Mathematics, 31(2): 197- 204, 2015.

[6] J.N. Flavin, R.J. Knops, Some Spatial Decay Estimates in Continuum Dynamics, J. Elasticity, 17: 249-264, 1987.

[7] A.E. Green, P.M. Naghdi, Thermoelasticity Without Energy Dissipation, J. Elasticity, 31: 189-208, 1993.

[8] A.E. Green, P.M. Naghdi, On Thermodynamics and the Nature of the Second Law, Proc. Roy. Soc. London A, 357: 253-270, 1977. 
[9] G. Groza, M. Jianu, N. Pop, Infinitely differentiable functions represented into Newton interpolating series, Carpathian Journal of Mathematics, 30(3): 309-316, 2014.

[10] L. Harabagiu, O. Simionescu-Panait, Propagation of inhomogeneous plane waves in isotropic solid crystals, Ann. Sci. Univ. Ovidius Constanta, 23 (3): 55-64, 2015

[11] D. Iesan, Thermal effects in orthotropic porous elastic beams, ZAMP, 60: 138-153, 2009.

[12] R. Kumar, Wave propagation in a microstretch thermoelastic diffusion solid, Ann. Sci. Univ. Ovidius Constanta, 23 (1): 127-169, 2015.

[13] M. Marin, On the minimum principle for dipolar materials with stretch, Nonlinear Analysis: Real World Applications, 10(3): 1572-1578, 2009

[14] M. Marin, G. Stan, Weak solutions in Elasticity of dipolar bodies with stretch, Carpathian Journal of Mathematics, 29(1): 33-40, 2013.

[15] M. Marin, R.P. Agarwal, S.R. Mahmoud, Modeling a microstretch thermoelastic body with two temperature, Abstr. Appl. Analysis, 2013: 1-7, 2031.

[16] M. Marin, O. Florea, On temporal behaviour of solutions in thermoelasticity of porous micropolar bodies, Ann. Sci. Univ. Ovidius Constanta, 22 (1): 169-188, 2014.

[17] R.D. Mindlin, Microstructure in linear Elasticity, Arch. Rational Mech. Anal., 16, 51-77, 1964

[18] L. Nappa, Spatial Decay Estimates for the Evolution Equations of Thermoelasticity Without Energy Dissipation, J. Thermal Stresses, 21: 581592, 1998.

[19] R. Quintanilla, On Existence in Thermoelasticity Without Energy Dissipation, J. Thermal Stresses, 25: 195-202, 2002.

[20] M. Marin, An evolutionary equation in thermoelasticity of dipolar bodies, Journal of Mathematical Physics, vol. 40 (3), 1391-1399, 1999

[21] M. Marin, R.P. Agarwal, S. R. Mahmoud, Nonsimple material problems addressed by the Lagrange's identity, Boundary Value Problems, vol. 2013, 1-14, 2013 art. no. 135. 
Marin MARIN,

Department of Mathematics and Computer Sciences,

Transilvania University of Brasov,

Bdul Iuliu Maniu, nr. 50, Brasov, Romania.

Email:m.marin@unitbv.ro

Ibrahim ABBAS,

Department of Mathematics, Faculty of Science \& Arts-Khulais,

King AbdulAziz University, 21384 Jeddah, Saudi Arabia.

Department of Mathematics,

Faculty of Science, Sohag University, 82524 Sohag, Egypt

Email: ibrabbas7@gmail.com 